\title{
REGULARIZATION FOR ACCURATE NUMERICAL WAVE PROPAGATION IN DISCONTINUOUS MEDIA*
}

\author{
ANNA-KARIN TORNBERG ${ }^{\dagger}$ AND BJÖRN ENGQUIST ${ }^{\ddagger}$
}

\begin{abstract}
Structured computational grids are the basis for highly efficient numerical approximations of wave propagation. When there are discontinuous material coefficients the accuracy is typically reduced and there may also be stability problems. In a sequence of recent papers Gustafsson et al. proved stability of the Yee scheme and a higher order difference approximation based on a similar staggered structure, for the wave equation with general coefficients. In this paper, the Yee discretization is improved from first to second order by modifying the material coefficients close to the material interface. This is proven in the $L^{2}$ norm. The modified higher order discretization yields a second order error component originating from the discontinuities, and a fourth order error from the smooth regions. The efficiency of each original method is retained since there is no special structure in the difference stencil at the interface. The main focus of this paper is on one spatial dimension, with the derivation of a second order algorithm for a two dimensional example given in the last section.
\end{abstract}

Key words. Yee scheme, discontinuous material coefficients, regularization

AMS subject classifications. 35L05, 35R05, 65M06, 65M12

1. Introduction. In a sequence of recent papers $[4,5,6]$, Gustafsson et al. developed and analyzed finite difference methods for wave equation approximations on structured and staggered grids. These algorithms were proved to be $L^{2}$-stable and well behaved even for discontinuous coefficients and of second and fourth order of numerical accuracy when the coefficients are regular. The second order discretization is the Yee scheme [15], and the fourth order discretization is as compact in time as the Yee scheme.

The main motivation to use this type of methods is computational efficiency. Stable higher order methods on unstructured grids based on finite element approximations are readily available and they can handle discontinuous material properties. Whenever it is possible to use the explicit structured grid techniques with staggered storage of the unknowns this is preferable because of efficiency. The gain in computational complexity and storage can often be more than an order of magnitude. This is why the Yee scheme in computational electromagnetics is still so popular in spite of its difficulty in handling discontinuous material properties and irregular boundaries $[10]$.

We have in a sequence of papers $[12,13,11]$ developed regularization techniques for computations with discontinuous coefficients and singular source terms. It is the purpose of the current work to apply these techniques to the numerical schemes of Gustafsson et al. [4, 5, 6], in order to produce a new and efficient class of methods. These new methods have similar stability properties as the original methods $[4,5]$ but are of higher order of accuracy also for discontinuous coefficients.

A major advantage of the algorithms we introduce here is that there is no special

\footnotetext{
*Received January 13, 2006; accepted for publication November 7, 2006.

$\dagger$ Courant Institute of Mathematical Sciences, New York University, 251 Mercer Street, New York, NY 10012, USA (tornberg@cims.nyu.edu). This work was partially sponsored by DOE Grant No DE-FG02-88ER25053

${ }^{\ddagger}$ Department of Mathematics, University of Texas, 1 University Station C1200, Austin, TX 78712, USA (engquist@math.utexas.edu). This work was partially supported by the Swedish Foundation for Strategic Research.
} 
structure in the difference stencil at the material interface. The same types of formulas are used all over the computational domain. The only components that are modified near the interfaces are the material coefficients of the original differential equations. This streamlines the computer code and makes rigorous proofs for stability and error estimates possible. An extension of this technology is to include boundary conditions that do not fit to the computational grid. It is covered in [14] and it is also based on adjusting the coefficients in the differential equations.

There have been other approaches to accommodate discontinuous material coefficients in finite difference approximations. Zhang and LeVeque [16] applied the immersed interface method to the acoustic wave equation. The Lax-Wendroff method (or a higher order scheme) was used away from the discontinuities, and a three point stencil (in 1D) was used closest to the discontinuities, with the weights derived using the physical jump conditions. In $2 \mathrm{D}$, a six point stencil was used at such irregular points. Ditkowski et al. [2] suggested a modification to the Yee scheme as applied to Maxwell's equations, which includes an extrapolation from grid values on the other side of the discontinuity, to the location of the discontinuity, and the use of this value in a modified stencil. In the case of a jump in the solution itself, this jump is taken into account in the modified stencil. This leads to a three point stencil for points closest to the discontinuity in 1D. Two other approaches to this problem, which both involve the introduction of "ghost" values close to the discontinuities can be found in $[9,8,17]$. Another popular approach is averaging of the discontinuous coefficients [1]. See also the review article [7] and the references therein.

The outline of this paper is as follows. In section 2, we give the differential equations and discuss the jump conditions that apply for the solutions at a point where the material properties are discontinuous. In section 3, we give the basic numerical schemes and the stability results, as derived in [4]. We proceed in section 4 to derive a regularization of the coefficients that yields a fully second order accurate scheme and we see that a specific regularization must be applied to the inverse of the coefficients. We prove first order convergence in $L^{2}$ for the Yee scheme with discontinuous coefficients, and second order convergence with the regularized coefficients. In section 5, this regularization technique is applied to a higher order discretization, resulting in a discretization with a second order error component arising from the discontinuities, and a fourth order error component from the smooth regions. In section 6 we consider a problem with a discontinuous solution. We indicate the multidimensional possibilities in the final section, where we derive the modifications needed to achieve second order accuracy for a selected example in two dimensions.

2. Equations and interface conditions. The wave equations approximated in $[4,5,6]$ have the form,

$$
\begin{aligned}
& p_{t}=a\left(u_{x}+v_{y}\right), \\
& u_{t}=b p_{x}, \\
& v_{t}=b p_{y},
\end{aligned}
$$

in two space dimensions and in one space dimension we have,

$$
\begin{aligned}
& p_{t}=a u_{x} \\
& u_{t}=b p_{x}
\end{aligned}
$$

The application discussed by Gustafsson et al. is acoustics with discontinuous material properties. The variable $p$ denotes pressure and $u, v$ the $x$ - and $y$-velocities 
respectively. The material coefficients are $a=-\rho c^{2}$ and $b=-1 / \rho$ with $\rho=\rho(x)$ density and $c=c(x)$ the speed of sound. We can naturally apply the techniques to other equations with the same structure as for example in electromagnetics, where the Maxwell's equations have the same structure as (1) in 2D [10].

In this paper, we are mainly concerned with the one-dimensional case, and we consider the system in Eq. (2). We assume that $a$ and $b$ are piecewise constant, discontinuous at $x=\bar{x}$, such that

$$
a(x)=\left\{\begin{array}{ll}
a^{L} & \text { if } x \leq \bar{x}, \\
a^{R} & \text { if } x>\bar{x},
\end{array} \quad b(x)= \begin{cases}b^{L} & \text { if } x \leq \bar{x} \\
b^{R} & \text { if } x>\bar{x} .\end{cases}\right.
$$

The solutions $u$ and $p$ are continuous across $\bar{x}$, whereas $u_{x}$ and $p_{x}$ and higher derivatives of $u$ and $p$ in general are not. Taylor expansion of $u(x, t)$ close to $\bar{x}$ yields

$$
u(x, t)= \begin{cases}u(\bar{x}, t)+\sum_{m=1}^{\infty} \frac{1}{m !}(x-\bar{x})^{m} \partial_{x}^{m} u^{L} & \text { if } x \leq \bar{x}, \\ u(\bar{x}, t)+\sum_{m=1}^{\infty} \frac{1}{m !}(x-\bar{x})^{m} \partial_{x}^{m} u^{R} & \text { if } x>\bar{x},\end{cases}
$$

where we have used the definitions

$$
\partial_{x}^{m} u^{L}=\lim _{x \rightarrow \bar{x}-} \partial_{x}^{m} u(x), \quad \partial_{x}^{m} u^{R}=\lim _{x \rightarrow \bar{x}+} \partial_{x}^{m} u(x) .
$$

The Taylor expansion of $p$ is on the same form.

Let us introduce the notation

$$
[f]=\lim _{x \rightarrow \bar{x}+} f(x)-\lim _{x \rightarrow \bar{x}-} f(x) .
$$

The solutions $u$ and $p$ are continuous, $[u]=[p]=0$. Therefore, $u_{t}$ and $p_{t}$ are also continuous, and from this fact, together with the system in (2), we obtain the jump conditions

$$
\begin{gathered}
{\left[a u_{x}\right]=a^{R} u_{x}^{R}-a^{L} u_{x}^{L}=0,} \\
{\left[b p_{x}\right]=b^{R} p_{x}^{R}-b^{L} p_{x}^{L}=0 .}
\end{gathered}
$$

3. The numerical schemes. The Yee scheme for the system in Eq. (2) is defined on a grid that is staggered both in space and time, with space step $h$ and time step $k$. The variables $p$ and $u$ are stored at half grid points $\left(x_{j+1 / 2}, t_{n+1 / 2}\right)$ and full grid points $\left(x_{j}, t_{n}\right)$ respectively, as shown in Figure 1 . Here, $x_{j}=j h, x_{j+1 / 2}=(j+1 / 2) h$, $t_{n}=n k$ and $t_{n+1 / 2}=(n+1 / 2) k$. With the difference operators defined by

$$
\begin{array}{ll}
D_{+} p_{j+1 / 2}=\left(p_{j+3 / 2}-p_{j+1 / 2}\right) / h, & D_{-} p_{j+1 / 2}=\left(p_{j+1 / 2}-p_{j-1 / 2}\right) / h, \\
D_{+} u_{j}=\left(u_{j+1}-u_{j}\right) / h, & D_{-} u_{j}=\left(u_{j}-u_{j-1}\right) / h,
\end{array}
$$

the Yee scheme [15] is

$$
\begin{aligned}
p_{j+1 / 2}^{n+1 / 2}-p_{j+1 / 2}^{n-1 / 2} & =k a_{j+1 / 2} D_{+} u_{j}^{n}, \\
u_{j}^{n+1}-u_{j}^{n} & =k b_{j} D_{-} p_{j+1 / 2}^{n+1 / 2} .
\end{aligned}
$$

The discretization error in space for the Yee scheme is $a h^{2} u_{x x x} / 24$ (evaluated at $x_{j+1 / 2}$ ) for the $p$-equation, and $b h^{2} p_{x x x} / 24$ (evaluated at $x_{j}$ ) for the $u$-equation. We have that

$$
\frac{p_{j+1 / 2}^{n+1 / 2}-p_{j+1 / 2}^{n-1 / 2}}{k}=p_{t}+\frac{k^{2}}{24} p_{t t t}+O\left(k^{4}\right)
$$




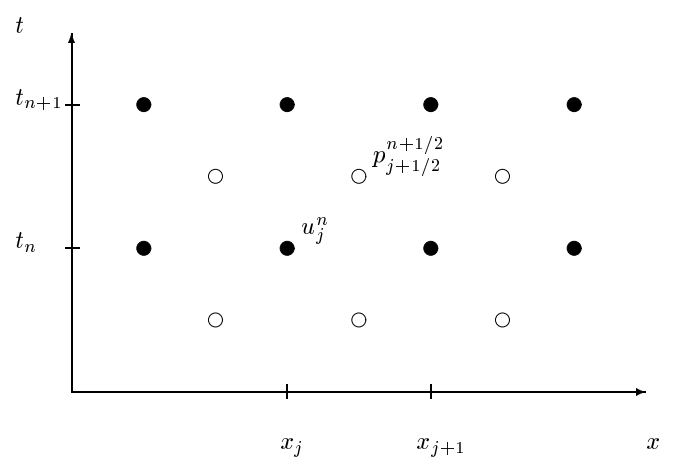

FIG. 1. The staggered grid

with $p_{t}$ and $p_{t t t}$ evaluated at $x_{j+1 / 2}, t_{n}$.

From the equations in (2),

$$
p_{t t}=\left(a u_{x}\right)_{t}=a u_{x t}=a u_{t x}=a\left(b p_{x}\right)_{x},
$$

and similarly $p_{t t t}=a\left(b\left(a u_{x}\right)_{x}\right)_{x}$. Also, $u_{t t t}=b\left(a\left(b p_{x}\right)_{x}\right)_{x}$. Using this to rewrite temporal derivatives to spatial derivatives and by subtracting off the second order error terms, as discretized to second order, a fourth order accurate scheme is obtained, see [4]. The scheme reads

$$
\begin{aligned}
p_{j+1 / 2}^{n+1 / 2} & =p_{j+1 / 2}^{n-1 / 2}+k a_{j+1 / 2} D_{+} u_{j}^{n}+\frac{k}{24} a_{j+1 / 2}\left(k^{2} D_{+} b_{j} D_{-} a_{j+1 / 2} D_{+}-h^{2} D_{+}^{2} D_{-}\right) u_{j}^{n}, \\
u_{j}^{n+1} & =u_{j}^{n}+k b_{j} D_{-} p_{j+1 / 2}^{n+1 / 2}+\frac{k}{24} b_{j}\left(k^{2} D_{-} a_{j+1 / 2} D_{+} b_{j} D_{-}-h^{2} D_{+} D_{-}^{2}\right) p_{j+1 / 2}^{n+1 / 2} .
\end{aligned}
$$

We will henceforth refer to this scheme as the $Q_{4}$ discretization.

These explicit schemes have $L^{2}$ stability conditions in form of time step restrictions $k \leq \lambda h$. The predicted CFL number $\lambda$ for the Yee scheme as given by the analysis in [4] is

$$
\lambda \sqrt{d_{1} d_{2}}<1
$$

where

$$
d_{1}=\max _{j} \frac{1}{2}\left(\sqrt{a_{j+1 / 2} b_{j}}+\sqrt{a_{j+1 / 2} b_{j+1}}\right), \quad d_{2}=\max _{j} \frac{1}{2}\left(\sqrt{a_{j+1 / 2} b_{j}}+\sqrt{a_{j-1 / 2} b_{j}}\right) .
$$

We can also define

$$
\bar{\lambda}=\max _{x} c(x) \cdot \lambda=\max _{x} \sqrt{a(x) b(x)} \cdot \lambda .
$$

This non-dimensionalized CFL number is independent of the scaling of the physical parameters. Naturally, $k \leq(\bar{\lambda} h) / \max _{x} c(x)$. For the fourth order scheme, the analysis in [4] yields

$$
\lambda \sqrt{d_{1} d_{2}}<\sqrt{\frac{12}{13+\beta}}
$$


with $d_{1}$ and $d_{2}$ as given above, and

$$
\beta=\max _{j}\left\{\max \left(\sqrt{a_{j+3 / 2} / a_{j+1 / 2}}, \sqrt{a_{j-1 / 2} / a_{j+1 / 2}}\right)\right\} .
$$

This is a sufficient condition for stability, together with the assumption

$$
\max _{j}\left[\max \left(\lambda^{2} a_{j+1 / 2} b_{j}, \lambda^{2} a_{j-1 / 2} b_{j}\right)\right]<2 .
$$

These restrictions on the time step size $k$ hold without any assumption regarding the smoothness of $a(x)$ and $b(x)$, and thus hold also for discontinuous coefficients.

4. Regularization for the Yee scheme. In the regularization technique [12], the material coefficients are modified in a narrow region close to the discontinuities in order to improve accuracy. An important property with this approach is that the structure of the basic scheme does not change. There are no special difference stencils at the interface and the stability analysis of the original scheme applies directly.

4.1. Derivation of regularized coefficients and error estimates. The local truncation error in the spatial discretization of the first equation in (2) as given by $a_{j+1 / 2} D_{+} u_{j}$ is $\left|a_{j+1 / 2} D_{+} u_{j}-a\left(x_{j+1 / 2}\right) u_{x}\left(x_{j+1 / 2}\right)\right|$. This is $O\left(h^{2}\right)$ away from the discontinuity.

For $x_{j} \leq \bar{x}<x_{j+1}$, we have that

$$
a_{j+1 / 2} D_{+} u_{j}=a_{j+1 / 2} \frac{u_{j+1}-u_{j}}{h}=\frac{1}{h} a_{j+1 / 2}\left(\left(x_{j+1}-\bar{x}\right) u_{x}^{R}-\left(x_{j}-\bar{x}\right) u_{x}^{L}\right)+O(h),
$$

where $u_{j+1}$ and $u_{j}$ have been Taylor expanded around $\bar{x}$, using the expansion in Eq. (4). Again using Taylor expansion around $\bar{x}, a\left(x_{j+1 / 2}\right) u_{x}\left(x_{j+1 / 2}\right)$ evaluates as

$$
a\left(x_{j+1 / 2}\right) u_{x}\left(x_{j+1 / 2}\right)= \begin{cases}a^{L}\left(u_{x}^{L}+\left(x_{j+1 / 2}-\bar{x}\right) u_{x x}^{L}\right)+O\left(h^{2}\right) & \text { if } x_{j+1 / 2} \leq \bar{x} \\ a^{R}\left(u_{x}^{R}+\left(x_{j+1 / 2}-\bar{x}\right) u_{x x}^{R}\right)+O\left(h^{2}\right) & \text { if } x_{j+1 / 2}>\bar{x} .\end{cases}
$$

Let us consider the error $\left|a_{j+1 / 2} D_{+} u_{j}-a\left(x_{j+1 / 2}\right) u_{x}\left(x_{j+1 / 2}\right)\right|$. As can be seen from above, keeping $a$ discontinuous yields an $O(1)$ error in this point. A similar analysis for $b_{j} D_{-} p_{j+1 / 2}$ yields an $O(1)$ error in the approximation of $b\left(x_{j}\right) p_{x}\left(x_{j}\right)$ for $\bar{x}-h / 2<x_{j} \leq \bar{x}+h / 2$. The Yee scheme is formally second order accurate. To achieve this second order accuracy we can typically allow for one order lower local truncation error at a few grid points. This is established in theorem 1 below. Compare also to the boundary condition case $[3,14]$. With these local $O(1)$ errors, the accuracy will however degenerate to first order in the approximations of both $u$ and $p$. This is intuitive, since the location of the discontinuity, $\bar{x}$, can be translated by up to $h / 2$ (remember the staggering) without any change in the numerical solution.

We can however improve the $O(1)$ local truncation error to $O(h)$ if let $a_{j+1 / 2}=$ $\tilde{a}\left(x_{j+1 / 2}\right)$, and define $\tilde{a}(x)$ such that we have

$$
\frac{1}{h} a_{j+1 / 2}\left(\left(x_{j+1}-\bar{x}\right) u_{x}^{R}-\left(x_{j}-\bar{x}\right) u_{x}^{L}\right)=a^{R} u_{x}^{R}=a^{L} u_{x}^{L},
$$

for any $\bar{x}$ such that $x_{j} \leq \bar{x}<x_{j+1}$. The equality $a^{L} u_{x}^{L}=a^{R} u_{x}^{R}$ comes from the jump condition (Eq. (6)). 
This yields that $\tilde{a}$ on the interval $[\bar{x}-h / 2, \bar{x}+h / 2]$ must be the inverse of a linear function, with value $1 / a^{L}$ at $\bar{x}-h / 2$ and value $1 / a^{R}$ at $\bar{x}+h / 2$. Hence,

$$
\tilde{a}(x)= \begin{cases}a^{L} & \text { if } x \leq \bar{x}-h / 2, \\ \Theta\left((x-\bar{x}) / h, a^{L}, a^{R}\right) & \text { if } \bar{x}-h / 2<x \leq \bar{x}+h / 2, \\ a^{R} & \text { if } x>\bar{x}+h / 2\end{cases}
$$

where

$$
\Theta\left(\eta, \phi^{L}, \phi^{R}\right)=\frac{\phi^{L} \phi^{R}}{\frac{1}{2}\left(\phi^{L}+\phi^{R}\right)+\eta\left(\phi^{L}-\phi^{R}\right)} .
$$

We could also write

$$
\tilde{a}(x)=\frac{1}{\frac{1}{a^{L}}+H_{h}(x-\bar{x})\left(\frac{1}{a^{R}}-\frac{1}{a^{L}}\right)},
$$

where $H_{h}(z)$ is the regularized Heaviside function

$$
H_{h}(z)= \begin{cases}0 & \text { if } z \leq-h / 2 \\ \frac{1}{2}+\frac{z}{h} & \text { if }-h / 2<z \leq h / 2 \\ 1 & \text { if } z>h / 2\end{cases}
$$

Hence, we have regularized 1/a rather than $a$ itself, using this regularized Heaviside function. A similar derivation can be done for the spatial discretization of the second equation in (2). This yields that the same regularization should be applied to $b(x)$, i.e. we define

$$
\tilde{b}(x)= \begin{cases}b^{L} & \text { if } x \leq \bar{x}-h / 2 \\ \Theta\left((x-\bar{x}) / h, b^{L}, b^{R}\right) & \text { if } \bar{x}-h / 2<x \leq \bar{x}+h / 2, \\ b^{R} & \text { if } x>\bar{x}+h / 2\end{cases}
$$

with the function $\Theta$ as defined in Eq. (19).

Applying the Yee scheme with these regularized coefficients will yield a first order truncation error in only a few points (one in each equation) close to the discontinuity, and a second order truncation error in all other points. Theorem 1 gives second order accuracy in $L^{2}$ and the numerical results indicate that this is also true in $L^{\infty}$.

Hence, the regularization we suggest for the Yee scheme in Eqs. (9)-(10) is to define

$$
a_{j+1 / 2}=\tilde{a}\left(x_{j+1 / 2}\right), \quad b_{j}=\tilde{b}\left(x_{j}\right),
$$

with $\tilde{a}(x)$ and $\tilde{b}(x)$ defined in Eq. (18) and Eq. (22), respectively.

Other regularizations, such as a linear regularization applied directly to $a$ instead of $1 / a$, will yield an $O(1)$ error in the spatial discretization in at least one grid point close to the discontinuity, as can be seen from the Taylor expansions above. This will yield first order error overall, albeit possibly with a smaller error coefficient than for the piecewise discontinuous coefficients.

In order to prove optimal convergence rate we need to show that the effect of the larger local truncation error is spread to a wider domain. Otherwise the order of accuracy estimate would be $O\left(h^{1 / 2}\right)$ weaker, see remark 4.1. We prove this by introducing an auxiliary problem in lemma 1 , and then comparing the numerical solution to the sum of the analytical solution and the solution of the auxiliary equations. In 
the auxiliary problem it is easier to prove that the truncation error, which in that problem corresponds to boundary values, will spread following the characteristics to a larger domain.

LEMMA 1. For any functions $f_{1}(t), f_{2}(t) \in C^{3}[0, T]$ there exist $\tilde{u}(x, t), \tilde{p}(x, t) \in$ $C^{3}(\mathbb{R} \backslash \bar{x},[0, T])$ such that $\tilde{u}, \tilde{p}$ are solutions to (2), (3) for $x \neq \bar{x}$ and

$$
\begin{aligned}
& \lim _{\varepsilon \rightarrow 0}(\tilde{u}(\bar{x}+\varepsilon, t)-\tilde{u}(\bar{x}-\varepsilon, t))=[\tilde{u}(\bar{x}, t)]=f_{1}(t), \\
& \lim _{\varepsilon \rightarrow 0}(\tilde{p}(\bar{x}+\varepsilon, t)-\tilde{p}(\bar{x}-\varepsilon, t))=[\tilde{p}(\bar{x}, t)]=f_{2}(t) .
\end{aligned}
$$

Proof. Extend the functions $f_{1}$ and $f_{2}$ to $[-\tau, T], \tau>0$, such that

$$
f_{1}, f_{2} \in C^{3}[-\tau, T], \quad f_{1}^{(k)}(-\tau)=f_{2}^{(k)}(-\tau)=0, k=0,1,2,3 .
$$

The functions $\tilde{u}(x, t), \tilde{p}(x, t)$ are defined as solutions to $(2),(3)$ in the domains $(-\infty<$ $x<\bar{x}$ ) and $\bar{x}<x<\infty)$ separately, with initial conditions $\tilde{u}=\tilde{p}=0$ at $t=-\tau$. The boundary conditions at $x=\bar{x}$ : $\tilde{u}_{+}(t), \tilde{u}_{-}(t), \tilde{p}_{+}(t), \tilde{p}_{-}(t)$, are given such that they satisfy the same conditions as in (26) and

$$
\lim _{\varepsilon \rightarrow 0} \tilde{u}(x \pm \varepsilon, t)=\tilde{u}_{ \pm}(t), \quad \lim _{\varepsilon \rightarrow 0} \tilde{p}(x \pm \varepsilon, t)=\tilde{p}_{ \pm}(t)
$$

Hence,

$$
\tilde{u}_{+}(t)-\tilde{u}_{-}(t)=f_{1}(t), \quad \tilde{p}_{+}(t)-\tilde{p}_{-}(t)=f_{2}(t) .
$$

The characteristics are found by diagonalizing the original wave equation. We define boundary conditions for the characteristic quantities emerging from the boundary $x=\bar{x}$,

$$
\tilde{p}_{-}+\alpha^{L} \tilde{u}_{-}=\beta^{L}, \quad \tilde{p}_{+}-\alpha^{R} \tilde{u}_{+}=\beta^{R}
$$

where $\alpha^{L}=\sqrt{a^{L} / b^{L}}$ and $\alpha^{R}=\sqrt{a^{R} / b^{R}}$. and the functions $\beta^{L}(t)$ and $\beta^{R}(t)$ remain to be determined. We also have the following homogeneous conditions due to the zero initial condition,

$$
\tilde{p}_{-}-\alpha^{L} \tilde{u}_{-}=0, \quad \tilde{p}_{+}+\alpha^{R} \tilde{u}_{+}=0 .
$$

The functions $\beta^{L}(t)$ and $\beta^{R}(t)$ are uniquely determined by the conditions above, together with condition (27). Hence, the necessary boundary conditions are known, and the solutions $\tilde{u}(x, t)$ and $\tilde{p}(x, t)$ are given by the method of characteristics and are in $C^{3}(\mathbb{R} \backslash x,[0, T])$.

THEOREM 1. Assume that p,u are compactly supported solutions of the wave equation (2) with $a, b$ defined in (3), such that $u, p \in C^{3}(\mathbb{R} \backslash \bar{x},[0, T])$. Let $p^{n+1 / 2}, u^{n}$ be solutions to the corresponding Yee scheme (9)-(10) with $k=\lambda h$, where $\lambda$ obeys the stability requirement in (13). The following error estimate is then valid $\left(0 \leq t^{n+1 / 2} \leq\right.$ $T)$,

$$
\left\|p\left(., t^{n+1 / 2}\right)-p^{n+1 / 2}\right\|_{L^{2}}+\left\|u\left(., t^{n}\right)-u^{n}\right\|_{L^{2}} \leq C h^{s},
$$

with $s=1$ for the Yee scheme with $a$ and $b$ defined by (3) and $s=2$ with $a$ and $b$ defined by (23). 
Proof. Consider first the Yee scheme (9)-(10), with unregularized coefficients (3), and assume $x_{m} \leq \bar{x}<x_{m+1 / 2}$. Let $p(x, t)$ and $u(x, t)$ be exact solutions to the wave equation. Using Taylor expansions, we get

$$
\begin{aligned}
& p\left(x_{j+1 / 2}, t^{n+1 / 2}\right)= p\left(x_{j+1 / 2}, t^{n-1 / 2}\right)+a_{j+1 / 2} k D_{+} u\left(x_{j}, t^{n}\right) \\
&+h g^{u}\left(t^{n}\right) \delta_{j, m}+\delta_{j m} O\left(h^{2}\right)+O\left(h^{3}\right), \\
& u\left(x_{j}, t^{n+1}\right)= u\left(x_{j}, t^{n}\right)+b_{j} k D_{-} p\left(x_{j+1 / 2}, t^{n+1 / 2}\right) \\
&+h g^{p}\left(t^{n+1 / 2}\right) \delta_{j, m}+\delta_{j m} O\left(h^{2}\right)+O\left(h^{3}\right), \\
& \text { where } \quad \delta_{j, m}= \begin{cases}1 & j=m, \\
0 & j \neq m .\end{cases}
\end{aligned}
$$

The last three terms in each equation comprise the scaled truncation error for this discretization. The local $O(h)$ term in the first equation, written as $h$ multiplying a $O(1)$ function $g^{u}$ occurs in relating $u_{x}\left(x_{j+1 / 2}\right)$ and $D_{+} u\left(x_{j}, t^{n}\right)$ for $x_{j}=x_{m}$, since $u_{x}$ is discontinuous at $x=\bar{x}$ (if $a^{R} / a^{L}=1$, then $u_{x}$ is continuous, and $g_{u}=0$ ). The function $g^{u}$ will depend on derivatives of $u$. Compare to the Taylor expansions in Eqs. (16)-(17) and the following discussion. The term $\mathrm{hg}^{p}$ occurs in a similar manner, as well as the local $O\left(h^{2}\right)$ terms.

We will define auxiliary functions $\tilde{p}(x, t)$ and $\tilde{u}(x, t)$ that will be used to match the terms $g^{u}$ and $g^{p}$. For the matching of the leading terms in the local truncation error, we will see below that we need,

$$
\begin{aligned}
\frac{a^{R} k}{h}[\tilde{u}(\bar{x}, t)] & =-g^{u}(t), \\
\frac{b^{L} k}{h}[\tilde{p}(\bar{x}, t)] & =-g^{p}(t) .
\end{aligned}
$$

This will be achieved with $f_{1}(t)=-\frac{h}{a^{R} k} g^{u}(t)$ and $f_{2}(t)=-\frac{h}{b^{L} k} g^{p}(t)$ in the Lemma. Since $g^{u}(t)$ and $g^{p}(t)$ are $O(1)$, so are $f_{1}(t)$ and $f_{2}(t)$, and hence also the auxiliary solutions $\tilde{u}(x, t)$ and $\tilde{p}(x, t)$.

Now, $\tilde{p}(x, t)$ and $\tilde{u}(x, t)$ have three continuous derivatives and satisfy the wave equation at each side of $\bar{x}$, but are discontinuous at $x=\bar{x}$. Using Taylor expansions, we now have the relation

$$
\tilde{u}_{x}\left(x_{m+1 / 2}, t\right)=D_{+} \tilde{u}\left(x_{m}, t\right)-\frac{[\tilde{u}(\bar{x}, t)]}{h}+O(1),
$$

and similarly for $p$. Hence, we have

$$
\begin{aligned}
\tilde{p}\left(x_{j+1 / 2}, t^{n+1 / 2}\right) & =\tilde{p}\left(x_{j+1 / 2}, t^{n-1 / 2}\right)+a_{j+1 / 2} k D_{+} \tilde{u}\left(x_{j}, t^{n}\right) \\
& +\frac{k}{h} a_{j+1 / 2} \delta_{j, m}\left\{-\left[\tilde{u}\left(\bar{x}, t^{n}\right)\right]+O(h)\right\}+O\left(h^{3}\right), \\
\tilde{u}\left(x_{j}, t^{n+1}\right) & =\tilde{u}\left(x_{j}, t^{n}\right)+b_{j} k D_{-} \tilde{p}\left(x_{j+1 / 2}, t^{n+1 / 2}\right) \\
& +\frac{k}{h} b_{j} \delta_{j, m}\left\{-\left[\tilde{p}\left(\bar{x}, t^{n+1 / 2}\right)\right]+O(h)\right\}+O\left(h^{3}\right) .
\end{aligned}
$$

Let us introduce,

$$
\begin{aligned}
\left(e^{p}\right)_{j+1 / 2}^{n+1 / 2} & =p\left(x_{j+1 / 2}, t^{n+1 / 2}\right)-h \tilde{p}\left(x_{j+1 / 2}, t^{n+1 / 2}\right)-p_{j+1 / 2}^{n+1 / 2}, \\
\left(e^{u}\right)_{j}^{n} & =u\left(x_{j}, t^{n}\right)-h \tilde{u}\left(x_{j}, t^{n}\right)-u_{j}^{n},
\end{aligned}
$$


where

$$
\left(e^{p}\right)_{j+1 / 2}^{1 / 2}=-h \tilde{p}\left(x_{j+1 / 2}, t^{1 / 2}\right)=O(h), \quad\left(e^{u}\right)_{j}^{0}=-h \tilde{u}\left(x_{j}, t^{0}\right)=O(h) .
$$

Using (28) for $p(x, t)$ and $u(x, t),(30)$ for $\tilde{p}(x, t)$ and $\tilde{u}(x, t)$, and the fact that $p_{j+1 / 2}^{n+1 / 2}$ and $u_{j}^{n}$ are solutions to the Yee scheme (9)-(10), we have

$$
\begin{gathered}
\left(e^{p}\right)_{j+1 / 2}^{n+1 / 2}=\left(e^{p}\right)_{j+1 / 2}^{n-1 / 2}+a_{j+1 / 2} k D_{+}\left(e^{u}\right)_{j}^{n}+\delta_{j, m} O\left(h^{2}\right)+O\left(h^{3}\right), \\
\left(e^{u}\right)_{j}^{n+1}=\left(e^{u}\right)_{j}^{n}+b_{j} k D_{-}\left(e^{p}\right)_{j+1 / 2}^{n+1 / 2}+\delta_{j, m} O\left(h^{2}\right)+O\left(h^{3}\right),
\end{gathered}
$$

The discrete error functions $e^{p}$ and $e^{u}$ satisfy the original difference equations modulo the order terms $\delta_{j, m} O\left(h^{2}\right)+O\left(h^{3}\right)$, and these terms have $L^{2}$ norms bounded by $C h^{5 / 2}$. The bound on the initial conditions and Duhamel's principle together with the basic discrete $L^{2}$ stability estimate then imply,

$$
\left\|\left(e^{p}\right)^{n+1 / 2}\right\|_{L^{2}} \leq C_{1} h, \quad\left\|\left(e^{u}\right)^{n}\right\|_{L^{2}} \leq C_{2} h
$$

and from the triangle inequality

$\left\|p\left(., t^{n+1 / 2}\right)-p^{n+1 / 2}\right\|_{L^{2}}+\left\|u\left(., t^{n}\right)-u^{n}\right\|_{L^{2}} \leq\left(C_{1}+C_{2}\right) h+h\left\|\tilde{p}^{n+1 / 2}\right\|_{L^{2}}+h\left\|\tilde{u}^{n}\right\|_{L^{2}} \leq C h$.

The case $x_{m-1 / 2} \leq \bar{x}<x_{m}$ can be handled in an analogous way.

The same argument also applies to the Yee scheme with modified coefficients (23). In this case, the terms $h g^{u}$ and $h g^{p}$ should be replaced by $h^{2} g^{u}$ and $h^{2} g^{p}$, where $g^{u}$ and $g^{p}$ are still $O(1)$ functions. Furthermore, the coefficients $a$ and $b$ in (29) should be appropriately modified. This yields the definition of $\left(e^{p}\right)_{j+1 / 2}^{n+1 / 2}$ and $\left(e^{u}\right)_{j}^{n}$ as in (31) but with $h^{2}$ in front of the $\tilde{p}$ and $\tilde{u}$ terms. These quantities now satisfy

$$
\begin{aligned}
\left(e^{p}\right)_{j+1 / 2}^{n+1 / 2} & =\left(e^{p}\right)_{j+1 / 2}^{n-1 / 2}+a_{j+1 / 2} k D_{+}\left(e^{u}\right)_{j}^{n}+O\left(h^{3}\right), \\
\left(e^{u}\right)_{j}^{n+1} & =\left(e^{u}\right)_{j}^{n}+b_{j} k D_{-}\left(e^{p}\right)_{j+1 / 2}^{n+1 / 2}+O\left(h^{3}\right) .
\end{aligned}
$$

The fact that the initial conditions $\left(e^{p}\right)_{j+1 / 2}^{1 / 2}$ and $\left(e^{u}\right)_{j}^{0}$ in $(32)$ are now $O\left(h^{2}\right)$, yields an error bounded by $C h^{2}$ when we conclude the proof as before.

REMARK 4.1. If we would not use the auxiliary functions $\tilde{p}, \tilde{u}$ but instead directly apply the stability estimate to $p\left(., t^{n+1 / 2}\right)-p^{n+1 / 2}$ and $u\left(., t^{n}\right)-u^{n}$ we would get the weaker result

$$
\left\|p\left(., t^{n+1 / 2}\right)-p^{n+1 / 2}\right\|_{L^{2}}+\left\|u\left(., t^{n}\right)-u^{n}\right\|_{L^{2}} \leq C h^{1 / 2} .
$$

This follows from the $L_{2}$ norm of the local truncation error,

$$
\left\|g\left(t^{n}\right) \delta_{j, m}\right\|_{L^{2}}=\left(h \sum_{j} g\left(t^{n}\right)^{2}\left(\delta_{j, m}\right)^{2}\right)^{1 / 2}=\left(h g\left(t^{n}\right)\right)^{1 / 2}=\left|g\left(t^{n}\right)\right| h^{1 / 2} .
$$

REMARK 4.2. The $O(h)$ error that originates from the interface is given by the terms $h \tilde{p}(x, t)$ and $h \tilde{u}(x, t)$ and they are bounded by $h \cdot$ const for all time ( $p \pm \alpha u$, $\alpha=\sqrt{a / b}$, is constant along characteristics). On the other hand, the $O\left(h^{2}\right)$ error that originates from the $O\left(h^{3}\right)$ terms in the local truncation errors grows linearly in time. 
REMARK 4.3. The proof does not directly extend to the $Q_{4}$ discretization in (11)(12) that is fourth order accurate for regular $a(x), b(x)$. Substantial modification of the proof will be needed, but we conjecture that it should hold that an $O\left(h^{s}\right)(s \leq 4)$ scaled local truncation error (compare to $h^{s} g^{u}$ and $h^{s} g^{p}$ in the Yee scheme) at a finite number of points in the $x$-direction contributes $O\left(h^{s}\right)$ to the total error. Since the $O\left(h^{s}\right)$ local truncation error occurs in more than one grid point a simple set of auxiliary functions is not enough.

In [5], Gustafsson and Wahlund perform a accuracy analysis for the case of unregularized discontinuous coefficients both for the Yee and the $Q_{4}$ discretizations. The analysis is performed in Fourier space based on harmonic waves, and the authors show that there is a $O(h)$ component of the error originating from the interface in both cases.

4.2. Numerical results. In this section, we present numerical results for the Yee scheme. We solve the problem in the domain $0 \leq x \leq 2 \pi, 0 \leq t \leq T$ and introduce two discontinuities, one at $x=\bar{x}$ and one at $x=\bar{x}+\pi$. That is, we define

$$
(\rho(x), c(x))= \begin{cases}\left(\rho^{R}, c^{R}\right) & \text { if } \bar{x}<x \leq \bar{x}+\pi \\ \left(\rho^{L}, c^{L}\right) & \text { otherwise }\end{cases}
$$

and from here, $a(x)=-\rho(x) c(x)^{2}, b(x)=-1 / \rho(x)$.

With $\omega=\omega_{0} c_{L}$, where $\omega_{0}$ is an integer, the solution is periodic in space. If $|\bar{x}-\pi / 2|<\delta$, then for $0 \leq t<\pi / 2-\delta$, on the interval $[0, \pi)$ we have a solution

$0 \leq x \leq \bar{x}:$

$$
\begin{aligned}
& p(x, t)=\sin \left(\omega\left(t-\frac{x-\bar{x}}{c_{L}}\right)\right)-\frac{\rho_{L} c_{L}-\rho_{R} c_{R}}{\rho_{L} c_{L}+\rho_{R} c_{R}} \sin \left(\omega\left(t+\frac{x-\bar{x}}{c_{L}}\right)\right), \\
& u(x, t)=\frac{1}{\rho_{L} c_{L}}\left[\sin \left(\omega\left(t-\frac{x-\bar{x}}{c_{L}}\right)\right)+\frac{\rho_{L} c_{L}-\rho_{R} c_{R}}{\rho_{L} c_{L}+\rho_{R} c_{R}} \sin \left(\omega\left(t+\frac{x-\bar{x}}{c_{L}}\right)\right)\right],
\end{aligned}
$$

$\bar{x}<x<\pi:$

$$
\begin{aligned}
& p(x, t)=\frac{2 \rho_{R} c_{R}}{\rho_{L} c_{L}+\rho_{R} c_{R}} \sin \left(\omega\left(t-\frac{x-\bar{x}}{c_{R}}\right)\right), \\
& u(x, t)=\frac{2}{\rho_{L} c_{L}+\rho_{R} c_{R}} \sin \left(\omega\left(t-\frac{x-\bar{x}}{c_{R}}\right)\right) .
\end{aligned}
$$

For our experiments, we set $\rho_{L}=1.0 / 1.8, \rho_{R}=1, c_{L}=0.87879, c_{R}=1$. The analytical solution on $[0, \pi)$ for both $u$ and $p$ with $\omega_{0}=16$ is shown in Figure 2 at time $t=0.12 \pi$ for $\bar{x}=\pi / 2$.

The Yee scheme is staggered not only in space, but also in time, i.e. the numerical approximation of $u$ is available at times $t_{n}=n k$, and $p$ at times $t_{n+1 / 2}=(n+1 / 2) k$. To initialize the problem, we assign the correct values to $u_{j}^{0}$ and $p_{j+1 / 2}^{-1 / 2}$ for all $j$.

In order to compare errors for different grid resolutions, we need to measure these errors at the same points in time. To have the numerical solutions available at common points in time, both for $u$ and $p$, we use a grid refinement factor of 3 . We solve the problem on $[0,2 \pi]$ with periodic boundary conditions.

Since the error depends on the relative location of $\bar{x}$ to the grid points, we will make a number of $(M)$ shifts of $\bar{x}$, compute the error in maximum norm over the 

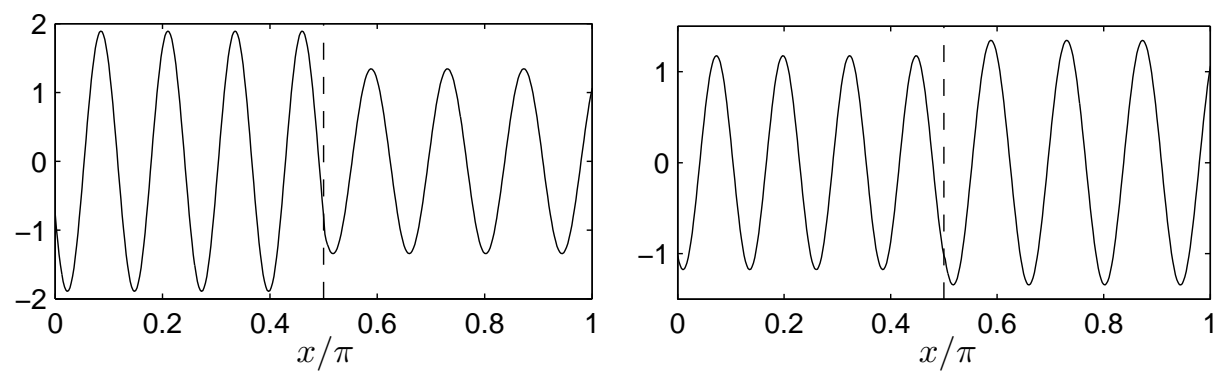

FIG. 2. The analytical solution of $u$ (to the left) and $p$ with $\omega_{0}=16$ is plotted versus $x / \pi$ on the interval $x \in[0, \pi)$ at $t=0.12 \pi(\bar{x}=\pi / 2)$.

interval $[0, \pi)$ for each, and then take the average. We let

$$
(\bar{x})_{l}=\left(\frac{1}{2}+\frac{\beta}{M} l h\right) \pi, \quad l=1, \ldots, M
$$

with $\beta=\sqrt{2} / 1.42$ an irrational number close to one. In the following we have used three grid resolutions, with the number of grid points $N=480,1440$ and 4320 . Hence, $h=2 \pi / N$, and we set $k=0.8 h$. The number of shifts $M=6$.

First, we keep $a$ and $b$ discontinuous. The error for $u$ and $p$ as a function of time, up to $t=0.48 \pi$ is shown in Figure 3.
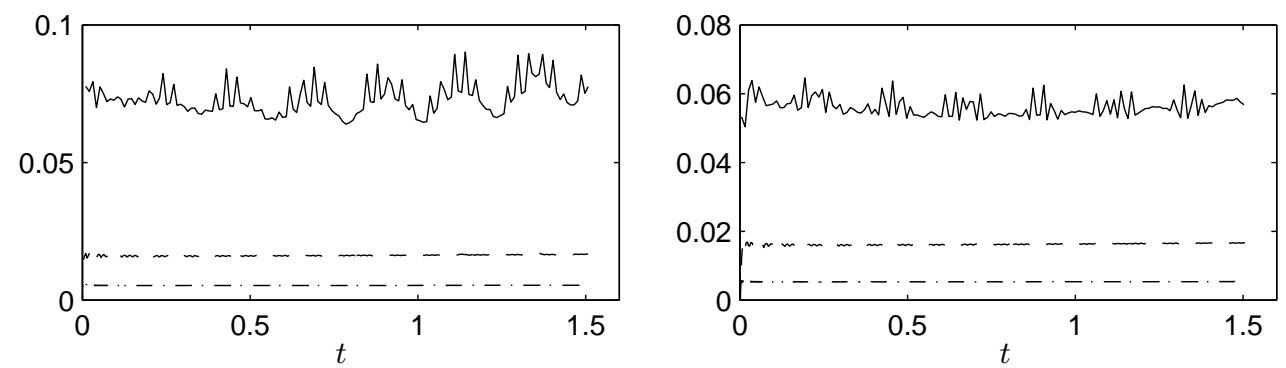

Fig. 3. Error in $u$ versus $t$ (to the left) and in $p$ versus $t$, for $N=480,1440$ and 4320, with $a$ and $b$ kept discontinuous. The errors have been measured in the maximum norm averaged over $M=6$ shifts of $\bar{x}$, as explained in connection to Eq. (36). $\omega_{0}=16$.
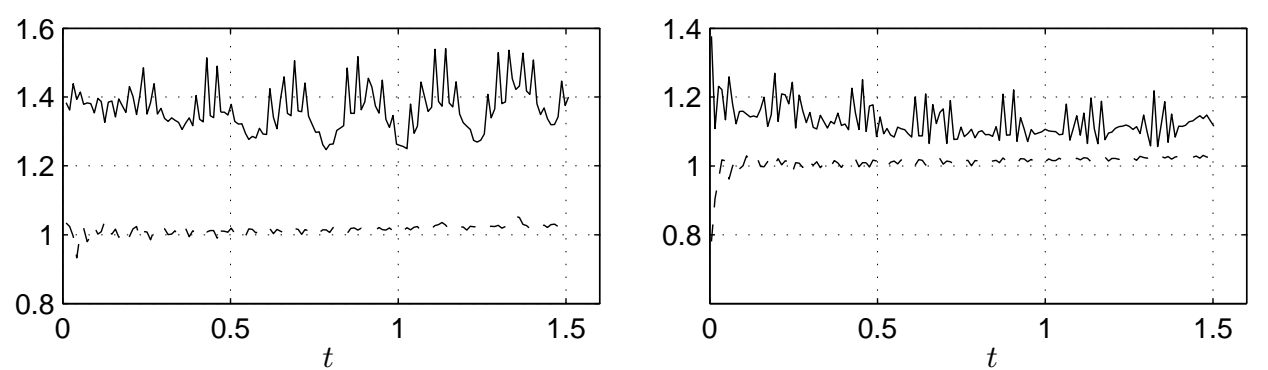

FIG. 4. The convergence rate for the numerical approximation of $u$ versus $t$ (to the left) and in $p$ versus $t$, as computed from the results displayed in Figure 3 (Based on the errors for $N=480$ and $N=1440$, solid line, and $N=1440$ and $N=4320$, dashed line). 
From the analysis, we expected a first order error. This is what we approach in the second refinement, which can be seen from the computed convergence rates in Figure 4. For the coarser grid, the first order error originating from the discontinuities does not as clearly dominate the second order error, yielding a computed convergence rate larger than one.

Now, let us repeat the same numerical experiment with $a$ and $b$ regularized as defined in Eq. (23). The error in the numerical approximations of $u$ and $p$ in this case is plotted versus time in Figure 5. Computing the convergence rates based on these
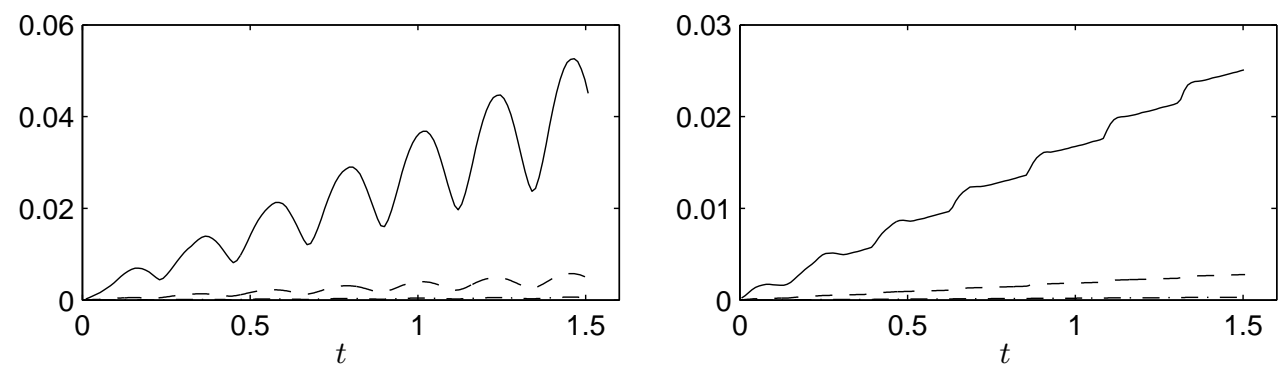

FIG. 5. Errors in $u$ (to the left) and in p, plotted versus t. As in Figure 3, but with $a$ and $b$ regularized as given in Eq. (23).

results, we find that for the first refinement, the convergence rate oscillates around 2 , as a function of time. For the second refinement, it is very close to the predicted second order at all times, as shown in Figure 6.
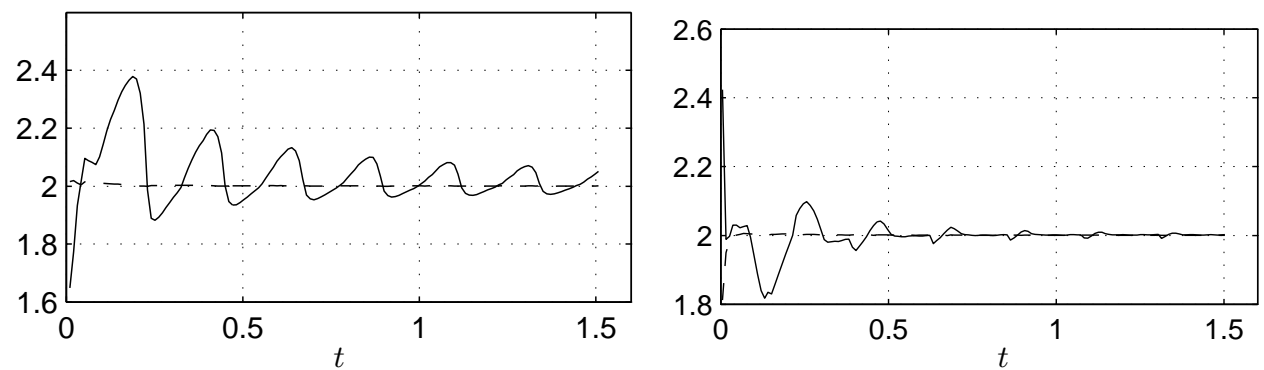

FIG. 6. The convergence rate for the numerical approximation of u versus $t$ (to the left) and in $p$ versus $t$, as computed from the results displayed in Figure 5 (Based on the errors for $N=480$ and $N=1440$, solid line, and $N=1440$ and $N=4320$, dashed line).

In Figure 5, the error is increasing with time. This is due to the second order frequency dependent error, that is present for the Yee scheme also with smooth coefficients. For unregularized coefficients, this error could not be seen in Figure 3, since it was obscured by the large first order error originating from the discontinuities.

Let us now consider a case with a larger $\omega$, i.e. with a more oscillatory solution. In Figure 7 we display the error up to $t=0.24 \pi$ for $\omega_{0}=128$, for $N=960,2880$ and 8640. These errors can be compared to the results in Figures 5, for $\omega_{0}=16$ (up to $t=0.48 \pi)$, which was produced for smaller $N$. The errors are of second order also for this larger $\omega$, but they are large at the end of the simulation, indeed very large for $N=960$. In Figure 8, the numerical solution for $N=960$ and the analytical solution is plotted in an interval around $\bar{x}$ at $t=0.12 \pi$. At this point, we can note the accumulating errors. For long time simulations, a second order method might not give 

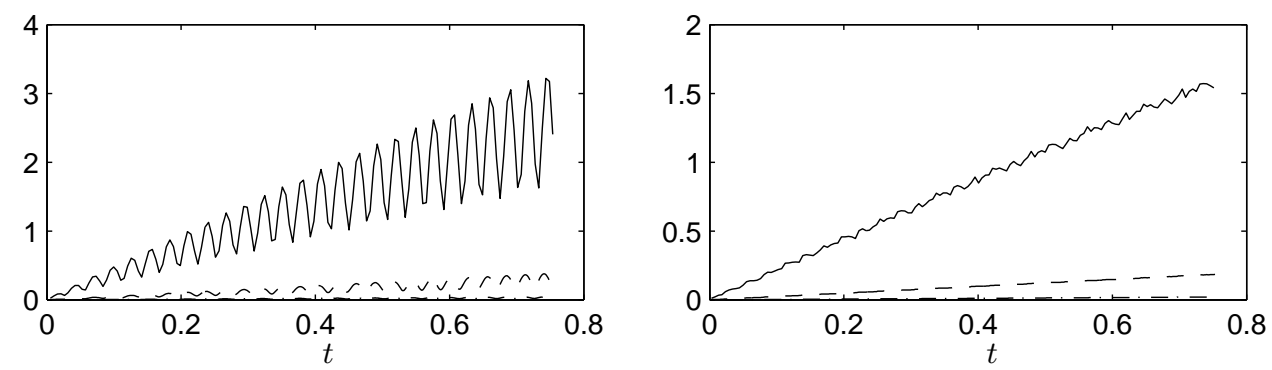

Fig. 7. Error in $u$ versus $t$ (to the left) and in $p$ versus $t$, with $\omega_{0}=128$ for $N=960,2880$ and 8640
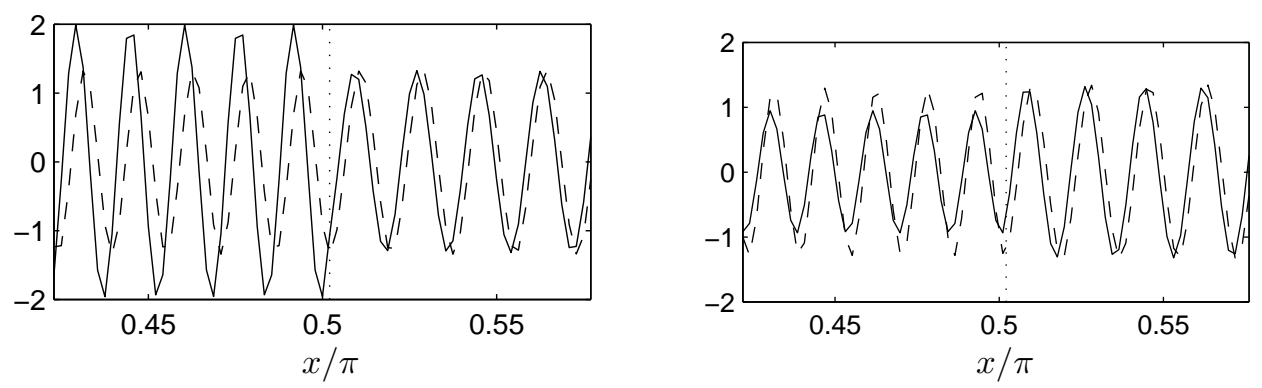

FIG. 8. The numerical solution (solid line) and analytical solution (dashed line) for $u$ and $p$ at $t=0.12 \pi$ for $\omega_{0}=128$, with $N=960$. Here, the accumulating errors can be clearly seen.

sufficient accuracy due to these errors, and it is often more efficient to use a higher order method, such as the $Q_{4}$ discretization introduced in (11)-(12). This method is fourth order accurate for smooth coefficients, and will be discussed in Section 5.

4.3. Numerical stability. The restriction on the non-dimensionalized CFL number $\bar{\lambda}$ sufficient for stability of the Yee scheme was given in Eqs. (13)-(14). We have tested this CFL condition in a range of experiments where the point wise initial values for $u$ and $p$ are given by random numbers between 0 and 1. This type of initial conditions will quickly cause any instability to grow. As in the numerical experiments described above, we let $\rho_{R}=c_{R}=1$ (i.e. $a_{R}=b_{R}=-1$ ) and $c_{L}=0.87879$, but we vary $\rho_{L}$. Hence, in these experiments, the CFL number $\lambda$ and the non-dimensionalized CFL number $\bar{\lambda}$ are the same.

With $\rho_{L}=0.001,0.1,33$ and 420 , the predicted maximum CFL numbers for the unregularized Yee scheme are 0.061, 0.49, 0.33 and 0.105 , respectively (as computed from Eq. (13)). The practical CFL numbers that we find by these experiments are very close to (slightly above) these theoretical limits. The CFL condition is the same for any shift of $\bar{x}$ in the grid, which can be seen from Eq. (13) together with the fact that there are two locations of discontinuitites: one at $x=\bar{x}$ where the coefficients are $\left(\rho_{L}, c_{L}\right)$ to the left and $\left(\rho_{R}, c_{R}\right)$ to the right, and one at $\bar{x}+\pi$ where the opposite is true.

For the regularized Yee scheme, as given in Eq. (23), the predicted maximum CFL numbers are again computed from Eq. (13), but now with the modified $a$ and $b$ coefficients. The predicted limits (minimum over $\bar{x}$ ) in the four cases mentioned above are $0.91,0.92,0.92$ and 0.91 .

In the numerical experiments, $\bar{\lambda}=0.99$ is stable in all cases, $\bar{\lambda}=1.0$ causes a 
slow growth of the numerical solution, and $\bar{\lambda}=1.01$ causes a rapid blow-up. The result is the same for all the many shifts in the grid that we have tested. Hence, it appears that the practical CFL limit is $\bar{\lambda}<1$ for the regularized scheme. This can be compared to the CFL numbers for the unregularized scheme - the lowest being $\bar{\lambda}<0.06$ for these cases.

It is apparent that, in addition to achieving a higher order accuracy with the regularized scheme, the stability properties of the scheme are also improved. Hence, it also results in a more efficient scheme, since larger time steps can be used.

4.4. An extreme test case. Before we conclude this section, let us consider a case with extreme physical contrasts, such as an interface between water and air. We set $\rho_{L}=1000, c_{L}=1500, \rho_{R}=1.3, c_{R}=340$. This yields the large ratios $a_{L} / a_{R}=14972, b_{L} / b_{R}=0.0013$.

In the expression for the analytical solution (Eqs.(34)-(35)), we can see that the magnitude of $u(x, t)$ is very small, due to the appearence of $\rho_{L} c_{L}$ in the denominator for the solution in both domains. For $p$, the reflection coefficient is close to one (0.9994) while the transmission coefficient is only $6 \cdot 10^{-4}$. The analytical solution for $p$ is plotted to the left in Figure 9.
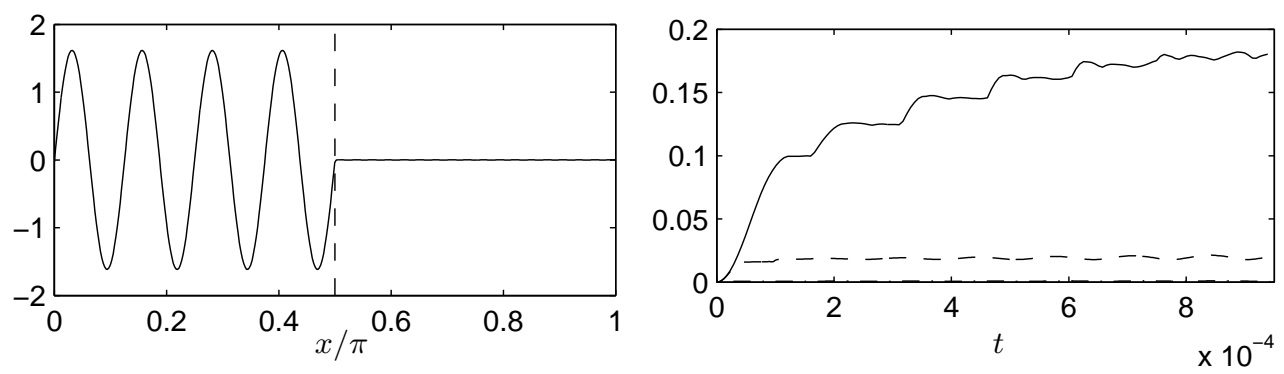

FIG. 9. The analytical solution of $p$ (to the left) for $\rho_{L}=1000, c_{L}=1500, \rho_{R}=1.3, c_{R}=340$ at $t=\left(3 \cdot 10^{-4}\right) \pi$. The solution is plotted versus $x / \pi$ on the interval $x \in[0, \pi)\left(\bar{x}=\pi / 2, \omega_{0}=16\right)$. The error for $p$ in the maximum norm is plotted versus time $t$ to the right for $N=480,1440$ and 4320 (averaged over $M=12$ shifts of $\bar{x}$ in the grid).

We regularize $a$ and $b$ as given in Eq. (23). For our numerical experiments, we again use a number of grid points of $N=480,1440$ and 4320 , with $M=12$ shifts (Eq. (36)) in the grid. The limit on the time step $k<(\bar{\lambda} h) /\left(\max _{x} c(x)\right)$, with $\bar{\lambda}=1$ as found in the previous section, evaluates as $k<\left(6.66 \cdot 10^{-4}\right) h$ due to the large wave speeds in the problem. We here set $k=\left(5 \cdot 10^{-4}\right) h$, which is stable for all choices of $\bar{x}$. We run the same number of time steps as before, up to $T=3 \cdot 10^{-4}$. The error in $p$ as a function of time is plotted to the right in figure 9. The error in $p$ is larger in this extreme case compared to the case that we considered before and the sensitivity of the error from the location of $\bar{x}$ is also larger. The largest errors occur when $\left(\bar{x}-x_{j}\right) / h=\theta \approx 0.7-0.8$, and is smaller when $\bar{x}$ is close either to a "full" grid point ( $\theta$ close to 0 or 1 ) or a "half" grid point ( $\theta$ close to 0.5$)$. We do however still obtain second order convergence (or even a bit higher). The error in $p$ for the finest resolution as plotted in the figure is less than $10^{-3}$ at the end time.

Without regularization, the Yee scheme is unstable for a time step of $k=(5$. $\left.10^{-5}\right) h$ (independent of $\bar{x}$ ), i.e. a time step that is ten times smaller than we have used with our regularized scheme. 
5. Higher order discretization. The $Q_{4}$ discretization in (11)-(12) is a fourth order accurate approximation to the system in (2), when the coefficients $a(x)$ and $b(x)$ are smooth. For this discretization, let us again consider the problem with discontinuities in $a$ and $b$ at $x=\bar{x}$, as defined in Eq. (3).

5.1. Derivation of regularization. In this work, we want to prescribe simple modifications to the coefficients $a$ and $b$, to improve the accuracy of the numerical scheme. The regularization should not depend on the numerical solution, such that it can be applied only once before initializing the time stepping. It does however not seem feasible to fully recover fourth order accuracy for the current discretization within this framework, the main difficulty being the correction term introduced to cancel the second order error term from the temporal discretization.

In Eq. (11), this correction term is $k^{2} D_{+} b_{j} D_{-} a_{j+1 / 2} D_{+} u_{j}^{n}$, and in in Eq. (12), this correction term is $k^{2} D_{-} a_{j+1 / 2} D_{+} b_{j} D_{-} p_{j+1 / 2}^{n+1 / 2}$. For smooth coefficients, these correction terms are of order $O\left(h^{2}\right)(k=\lambda h)$. However, for discontinuous $a$ and $b$, these correction terms will be $O(1)$. This is true also if we regularize the coefficients locally (over a region with the width proportional to $h$ ). Hence, this will limit the order of accuracy of the discretization to first order.

To see this, consider $k^{2} D_{+} b_{j} D_{-} a_{j+1 / 2} D_{+} u_{j}^{n}$, which approximates $\left(b(a u)_{x}\right)_{x}$, for an index $j$ such that $x_{j} \leq \bar{x}<x_{j+1}$. In the discretization, this term is multiplied by $a_{k+1 / 2}$ and a factor of $k^{2}$. Expanding $\left(b(a u)_{x}\right)_{x}$, we see that we have one term including $b_{x} a_{x}$ and one term including $b a_{x x}$. When $a$ and $b$ are either not regularized, or regularized over a region proportional to $h, a$ and $b$ will change by a constant over an interval of length $h$, and the numerical difference approximating the derivative will be proportional to $1 / h$. Hence, with two derivatives, we have a leading term which is proportional to $1 / h^{2}$. This term is multiplied by $k^{2}$, so with $k=\lambda h$, this term is $O(1)$. Hence, this will yield a method with a first order error component originating from the discontinuities.

Clearly, we want to do better than this. It is however of value to use the $Q_{4}$ discretization even if the error due to the discontinuities is of lower order. In [5], it was shown that with discontinuous coefficients, the error of the $Q_{4}$ scheme has one first order component originating from the discontinuities, and a fourth order error component that would be present also for smooth coefficients. This fourth order frequency dependent error can be kept small to a lower computational cost for long time integrations compared to the Yee scheme, for which this error is of second order. Hence, also in this case, a substantial reduction in the total error can can be achieved by using the $Q_{4}$ discretization compared to the Yee scheme [5].

Our aim here is to design a method with second order accuracy over all, but where the frequency dependent errors still are of fourth order. To obtain a second order error, we can regularize $a$ and $b$ as was done for the Yee scheme, and simply not add the correction terms in the $Q_{4}$ scheme in grid points close to the singularity. To formalize this, we write our modified $Q_{4}$ scheme as

$$
\begin{aligned}
p_{j+1 / 2}^{n+1 / 2} & =p_{j+1 / 2}^{n-1 / 2}+k a_{j+1 / 2} D_{+} u_{j}^{n} \\
& +\frac{k}{24} a_{j+1 / 2}\left(\alpha_{j+1 / 2} k^{2} D_{+} b_{j} D_{-} a_{j+1 / 2} D_{+}-\beta_{j+1 / 2} h^{2} D_{+}^{2} D_{-}\right) u_{j}^{n}, \\
u_{j}^{n+1} & =u_{j}^{n}+k b_{j} D_{-} p_{j+1 / 2}^{n+1 / 2} \\
& +\frac{k}{24} b_{j}\left(\alpha_{j} k^{2} D_{-} a_{j+1 / 2} D_{+} b_{j} D_{-}-\beta_{j} h^{2} D_{+} D_{-}^{2}\right) p_{j+1 / 2}^{n+1 / 2},
\end{aligned}
$$


where $\alpha(x) \equiv 1$ and $\beta(x) \equiv 1$ in the original $Q_{4}$ scheme.

The modified discretization described above is then defined by Eqs. (37)-(38), with

$$
\begin{aligned}
a_{j+1 / 2} & =\tilde{a}\left(x_{j+1 / 2}\right), \quad b_{j}=\tilde{b}\left(x_{j}\right), \\
\alpha(x) & =\beta(x)= \begin{cases}1 & \text { if }|x-\bar{x}| \geq 3 h / 2, \\
0 & \text { if }|x-\bar{x}|<3 h / 2 .\end{cases}
\end{aligned}
$$

with $\tilde{a}(x)$ and $\tilde{b}(x)$ as defined in Eq. (18) and Eq. (22), respectively.

The regularizations $\tilde{a}(x)$ and $\tilde{b}(x)$ were derived to improve on the order of accuracy of the spatial difference formulas $a_{j+1 / 2} D_{+} u_{j}$ and $b_{j} D_{-} p_{j+1 / 2}$, respectively. In the first equation of the $Q_{4}$ scheme (Eq. (11)), the term from the Yee scheme plus the correction term for the spatial error expands as

$$
a_{j+1 / 2}\left\{D_{+} u_{j}-\frac{h^{2}}{24} D_{+}^{2} D_{-} u_{j}\right\}=\frac{1}{24} \frac{1}{h} a_{j+1 / 2}\left\{-u_{j+2}+27 u_{j+1}-27 u_{j}+u_{j-1}\right\},
$$

which for smooth functions is a fourth order approximation to $a\left(x_{j+1 / 2}\right) u_{x}\left(x_{j+1 / 2}\right)$. Similarly, the term from the Yee scheme plus the correction term for the spatial error in Eq. (12) approximates $b\left(x_{j}\right) p_{x}\left(x_{j}\right)$ to fourth order for smooth coefficients.

Using Taylor series expansions on each side of $\bar{x}$ in the wider stencil above, similarly to what was done for the Yee discretization, we can derive the regularizations of $a$ and $b$ that matches this discretization such as to give a first order error at the grid points closest to the discontinuity. We obtain regularized functions that could then be used in the discretization (37)-(38), but this time with $\beta \equiv 1$ since the spatial correction terms should be included at each point.

However, the regularization we find this way will not yield a scheme with good properties for all choices of $a_{L}, a_{R}, b_{L}$ and $b_{R}$. As a matter of fact, the regularization is not even well defined for all sets of coefficients. Also for choices of coefficients where it is well defined, the stability properties of this scheme is inferior to that with the Yee regularization suggested in (39). Since the accuracy of the two schemes are comparable, we will not use this second regularization.

5.2. Numerical results. We now repeat the numerical experiments presented in Section 4. Before we introduce any regularization, we first present the error in the numerical solution for $u$ and $p$ when $a$ and $b$ are kept discontinuous. This is done in Figure 10 for $\omega_{0}=16$, and for $N=480,1440$ and 4320. The results can be compared to the ones for the Yee scheme, with no regularization applied, as presented in Figure 3.

Here, we have a first order error from the discontinuity (remember the factor of 3 in the refinement of the grid), and the total error is rather similar to the error for the Yee scheme without any regularization.

We now apply our $Q_{4}$ discretization with regularized coefficients (Eqs. (37)-(39)). The errors for $u$ and $p$ versus time are shown in Figure 11 and the computed convergence rates based on these results in Figure 12. The errors are much smaller than for the unregularized discretization, and the computed convergence rate for the second refinement is very close to second order at all times. These errors can be compared to the error for the Yee-scheme with the same regularization, as shown in Figure 5. In both cases, the errors are of second order, but for the Yee scheme, they are larger and growing in time. This is due to the fact that the second order error from the discontinuity blends with the second order frequency dependent error that grows in 

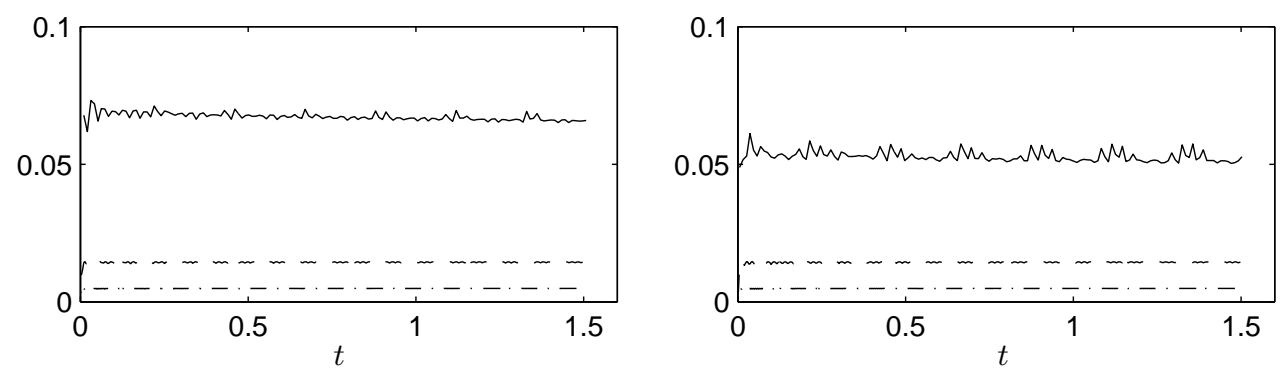

Fig. 10. Error in $u$ versus $t$ (to the left) and in $p$ versus $t$, for $N=480,1440$ and 4320 . The fourth order scheme in Eqs. (11)-(12) has been used, with a and b kept discontinuous. The error has been computed as the average of the error over $M=6$ shifts in $\bar{x}$ (Eq. (36)). For each shift the error has been computed in max norm over the grid points in the interval $[0, \pi)$.
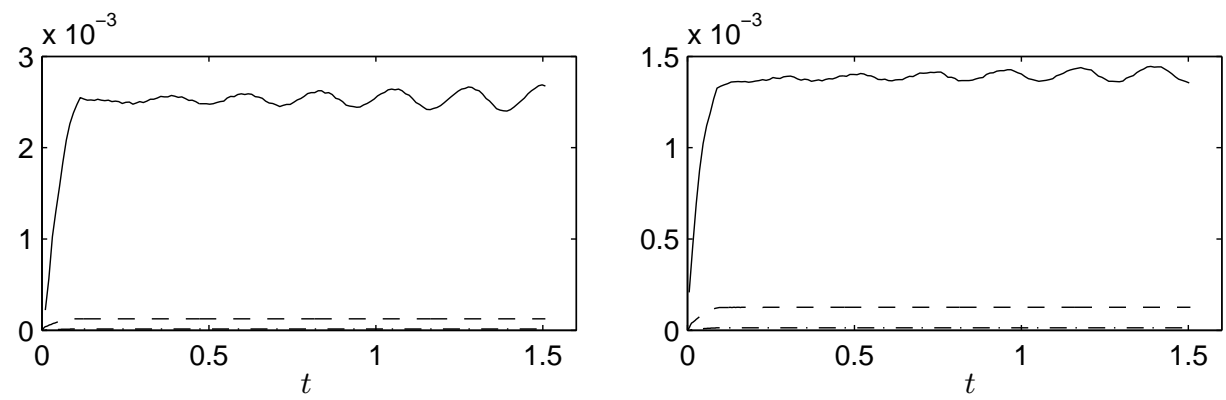

Fig. 11. Error in $u$ versus $t$ (to the left) and in $p$ versus $t$, for $N=480,1440$ and 4320 . As in figure 10, but with the regularization as given in Eq. (39) applied.

time. For the $Q_{4}$ discretization, the error component that would arise also for smooth coefficients is of fourth order, which for these grid resolutions and choice of $\omega_{0}$ is completely dominated by the second order error term arising from the treatment of the discontinuity. Hence, we can see no growth in time for the error.
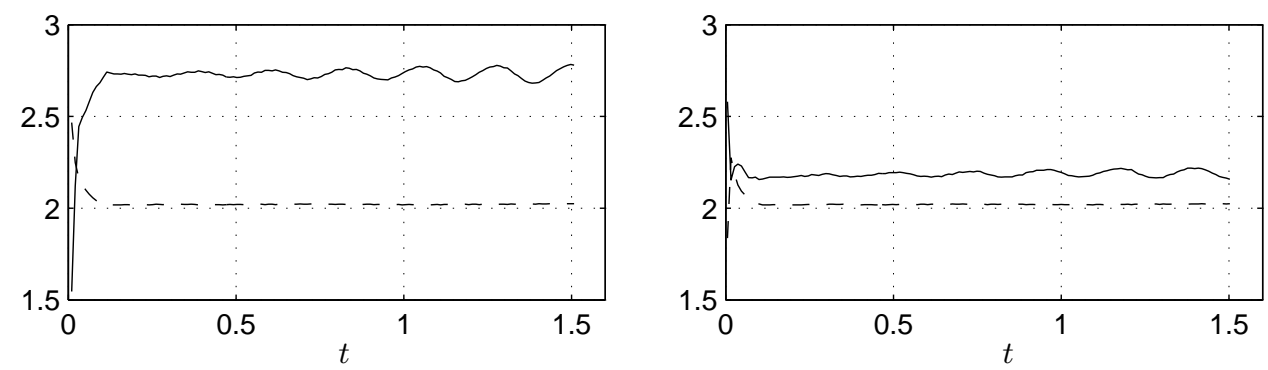

FIG. 12. The convergence rate for the numerical approximation of $u$ versus $t$ (to the left) and in $p$ versus $t$, as computed from the results in Figure 11.(Based on the errors for $N=480$ and $N=1440$, solid line, and $N=1440$ and $N=4320$, dashed line).

We now turn to the example with a more oscillatory solution, with $\omega_{0}=128$. For the Yee method, the results were displayed in Figure 7. We now repeat the same simulations, with our $Q_{4}$ discretization with regularized coefficients (Eqs. (37)-(39)). In Figure 13 we display the errors up to $t=0.24 \pi$ for $N=960,2880$ and 8640 . The convergence rates for these errors are computed to be higher than two (highest for the coarser grids), which indicates that the second order error does not as strongly 

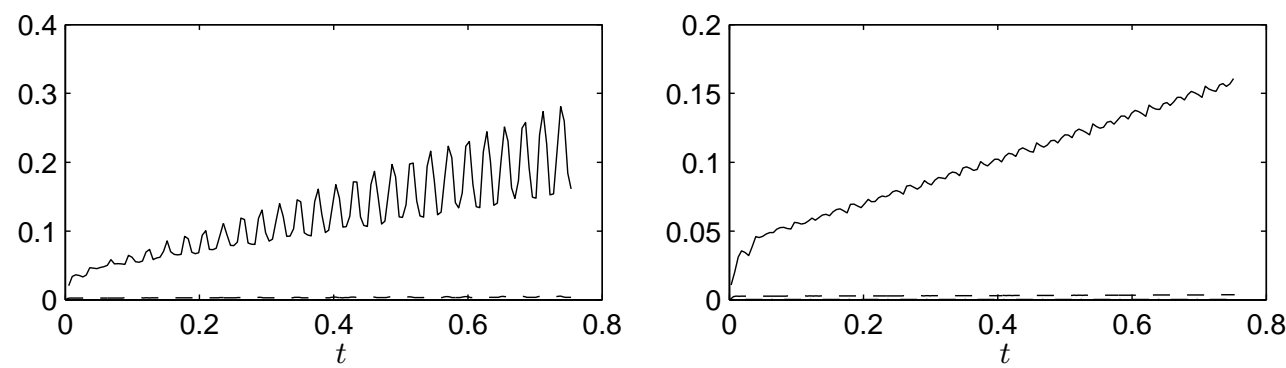

Fig. 13. Error in $u$ versus $t$ (to the left) and in $p$ versus $t$, for $N=960,2880$ and 8640 for $\omega_{0}=128$. The regularization as suggested in Eq. (39) has been applied.

dominate the fourth order error any more. That can be seen also from the fact that the total error grows with time, especially for the lowest value of $N$, in Figure 13.

The errors are however much smaller than the errors for the Yee scheme in Figure 7, where the frequency dependent errors are also of second order. Already for $N=960$, the errors for the $Q_{4}$ scheme is about ten times smaller than the errors for the Yee scheme, and this difference magnifies with consecutive refinements. Hence, even though the discontinuity is treated to second order (with regularization) for both schemes, and both schemes therefore are formally second order, it is in many cases computationally more efficient to use the $Q_{4}$ scheme, since a comparable total error can be obtained with a coarser grid and hence also a larger time step.

5.3. Numerical stability. The restriction on the non-dimensionalized CFL number $\bar{\lambda}$ sufficient for stability for the $Q_{4}$ scheme was given in Eqs. (15) together with (14). This can be applied directly to the unregularized scheme. With $\rho_{R}=c_{R}=1$ (i.e. $a_{R}=b_{R}=-1$ ), $c_{L}=0.87879$, and a varying $\rho_{L}$ of $\rho_{L}=0.001,0.1,33$ and 420 , the predicted maximum CFL numbers for the unregularized fourth order scheme are $0.03,0.41,0.27$ and 0.065 , respectively. Compare to section 4.3 for results for the Yee scheme.

For the regularized scheme as given in Eqs. (37)-(39), the theory does not directly apply, due to the fact that we mask out the correction terms at a few points close to the discontinuity. For a stability proof with a masking of a somewhat different form, see [14]. For the $Q_{4}$ scheme with the regularized $a$ and $b$ but without masking of correction terms, the predicted CFL limits (minimum over $\bar{x}$ ) are about $\bar{\lambda}=0.45$, $0.78,0.78$ and 0.60 , respectively, for the different values of $\rho_{L}$ listed above. However, close to the discontinuity (which is where the coefficients vary), the scheme is the regularized Yee scheme, for which the predicted limits on $\bar{\lambda}$ is closer to 1 .

We perform the same numerical experiments as in Section (4.3), and find the same results as for the regularized Yee scheme. That is, we find a practical CFL limit of $\bar{\lambda}<1$, independent of $\bar{x}$. Hence, it appears as if the addition of the correction terms away from the discontinuity, to achieve fourth order accuracy in the domain, does not alter the stability limit compared to the regularized Yee scheme.

Even though the practical CFL limits that we find for the unregularized $Q_{4}$ scheme is higher than the predicted limits - about 0.06 instead of 0.03 for $\rho=0.001$ for example, they are much smaller than the limit of $\bar{\lambda}<1$ for the regularized scheme. Hence, the regularized scheme is not only more accurate, but also more efficient than the unregularized scheme, since larger time steps can be used. 
6. A problem with discontinuous solution. Consider the system

$$
\begin{aligned}
& p_{t}=(a u)_{x}, \\
& u_{t}=b p_{x} .
\end{aligned}
$$

We again assume that $a$ and $b$ are piecewise constant, discontinuous at $x=\bar{x}$, as given in Eq. (3).

In the previous example, for the system in Eq. (2), the solutions $u$ and $p$ are continuous across $\bar{x}$, whereas $u_{x}$ and $p_{x}$ are not. In this case, $u$ itself is discontinuous across $\bar{x}$. The understanding of this discontinuous case is important for approximations in higher dimensions, see section 7 . With $\tilde{u}=-a u$, we can write (40) as

$$
\begin{aligned}
& p_{t}=-\tilde{u}_{x}, \\
& \tilde{u}_{t}=-(a b) p_{x} .
\end{aligned}
$$

This is the system we have already considered, for which we know the jump conditions (Eq. (6)). Hence, for the solutions to (40) it holds

$$
\begin{gathered}
{[a u]=a^{R} u^{R}-a^{L} u^{L}=0, \quad\left[(a u)_{x}\right]=a^{R} u_{x}^{R}-a^{L} u_{x}^{L}=0,} \\
{[p]=p^{R}-p^{L}=0, \quad\left[(a b) p_{x}\right]=a^{R} b^{R} p_{x}^{R}-a^{L} b^{L} p_{x}^{L}=0 .}
\end{gathered}
$$

6.1. Modified coefficients. Consider the system in Eq. (40) as discretized by the second order method,

$$
\begin{gathered}
p_{j+1 / 2}^{n+1 / 2}=p_{j+1 / 2}^{n-1 / 2}+k D_{+}\left(a_{j} u_{j}^{n}\right), \\
u_{j}^{n+1}=u_{j}^{n}+k b_{j} D_{-} p_{j+1 / 2}^{n+1 / 2} .
\end{gathered}
$$

The error in the spatial discretization of the first equation in (40) is given by $\left|D_{+}\left(a_{j} u_{j}\right)-\left(a\left(x_{j+1 / 2}\right) u\left(x_{j+1 / 2}\right)\right)_{x}\right|$. This is $O\left(h^{2}\right)$ away from the discontinuity.

For $x_{j} \leq \bar{x}<x_{j+1}$, we have that

$$
\frac{a_{j+1} u_{j+1}-a_{j} u_{j}}{h}=\frac{1}{h}\left(a_{j+1}\left(u^{R}+\left(x_{j+1}-\bar{x}\right) u_{x}^{R}\right)-a_{j}\left(u^{L}+\left(x_{j}-\bar{x}\right) u_{x}^{L}\right)\right)+O(h),
$$

where $u_{j+1}$ and $u_{j}$ have been Taylor expanded around $\bar{x}$, using the expansion in Eq. (4). Again using Taylor expansion around $\bar{x},(a u)_{x}$ at $x_{j+1 / 2}$ with $a$ piecewise constant evaluates as

$$
\left.(a u)_{x}\right|_{x_{j+1 / 2}}=a\left(x_{j+1 / 2}\right) u_{x}\left(x_{j+1 / 2}\right)= \begin{cases}a^{R} u_{x}^{R}+O(h) & \text { if } \bar{x} \leq x_{j+1 / 2}, \\ a^{L} u_{x}^{L}+O(h) & \text { if } \bar{x}>x_{j+1 / 2} .\end{cases}
$$

Using the jump conditions, we have that $a^{R} u^{R}=a^{L} u^{L}$ and $a^{R} u_{x}^{R}=a^{L} u_{x}^{L}$, and hence, if we let $a_{j+1}=a^{R}$ and $a_{j}=a^{L}$, from Eq. (45), we get $\left(a_{j+1} u_{j+1}-a_{j} u_{j}\right) / h=$ $a^{R} u_{x}^{R}+O(h)=a^{L} u_{x}^{L}+O(h)$. Hence, without any modification of $a$, we obtain a first order error in this one point. Actually, if we would introduce any regularization such that $a_{j+1} u^{R} \neq a_{j} u^{L}$, there will be an $O(1 / h)$ error in this spatial discretization in one point, and hence a potential $O(1)$ error in the numerical solutions to the system.

Let us now consider the second equation, for $x_{j-1 / 2} \leq \bar{x}<x_{j+1 / 2}$, where $b_{j}\left(p_{j+1 / 2}-p_{j-1 / 2}\right) / h$ should approximate $b\left(x_{j}\right) p_{x}\left(x_{j}\right)$. Using the same kind of Taylor expansions, and the fact that $p$ is continuous, yields that to obtain a first order error we must require

$$
\frac{b_{j}}{h}\left(\left(x_{j+1 / 2}-\bar{x}\right) p_{x}^{R}+\left(x_{j-1 / 2}-\bar{x}\right) p_{x}^{L}\right)= \begin{cases}b^{L} p_{x}^{L} & \text { if } x_{j}<\bar{x} \\ b^{R} p_{x}^{R} & \text { if } x_{j}>\bar{x} .\end{cases}
$$


The jump condition is $a^{R} b^{R} p_{x}^{R}=a^{L} b^{L} p_{x}^{L}$. Hence, if $x_{j}<\bar{x}$, we replace $p_{x}^{R}=$ $\left(a^{L} b^{L}\right) /\left(a^{R} b^{R}\right) p_{x}^{L}$ in the expression to the left. Then we can divide by $p_{x}^{L}$ on both sides, and obtain an expression for $b_{j}$. Similarly, if $x_{j}>\bar{x}$, we replace $p_{x}^{L}=\left(a^{R} b^{R}\right) /\left(a^{L} b^{L}\right) p_{x}^{R}$. We obtain the regularized, or rather modified, function

$$
\hat{b}(x)= \begin{cases}b^{L} & \text { if } x \leq \bar{x}-h / 2 \\ \Theta\left((x-\bar{x}) / h, a^{L} b^{L}, a^{R} b^{R}\right) / a^{L} & \text { if } \bar{x}-h / 2<x<\bar{x} \\ \Theta\left((x-\bar{x}) / h, a^{L} b^{L}, a^{R} b^{R}\right) / a^{R} & \text { if } \bar{x}<x<\bar{x}+h / 2 \\ b^{R} & \text { if } x \geq \bar{x}+h / 2\end{cases}
$$

where

$$
\Theta\left(\eta, \phi^{L}, \phi^{R}\right)=\frac{\phi^{L} \phi^{R}}{\frac{1}{2}\left(\phi^{L}+\phi^{R}\right)+\eta\left(\phi^{L}-\phi^{R}\right)} .
$$

We have written it in this manner to relate this to the inverse linear regularization that we derived for $a$ and $b$ in Section 4 . In this case, to define the modification for $b$, we apply the inverse linear regularization to the product $a b$, where after we divide by the discontinuous $a$. Hence, the modified $b$ is in this case discontinuous.

Hence, the modification we suggest for the scheme in Eqs. (43)-(44) is to define

$$
a_{j+1 / 2}=a(x), \quad b_{j}=\hat{b}\left(x_{j}\right),
$$

with the discontinuous $a(x)$ defined in Eq. (3) and the modified $\hat{b}(x)$ in Eq. (46). We expect this discretization to give second order accuracy, also at the grid points closest to the discontinuity.

6.2. Numerical results. Again, we solve the problem in the domain $0 \leq x \leq$ $2 \pi, 0 \leq t \leq T$. We introduce two discontinuities, one at $x=\bar{x}$ and one at $x=\bar{x}+\pi$. That is, we define

$$
(a(x), b(x))= \begin{cases}\left(a^{R}, b^{R}\right) & \text { if } \bar{x}<x \leq \bar{x}+\pi \\ \left(a^{L}, b^{L}\right) & \text { otherwise. }\end{cases}
$$

with $a^{L}=-1 / 2, a^{R}=-1, b^{L}=-1.8$ and $b^{R}=-1$.

In the above, the system in Eq. (40) was by a simple rescaling rewritten as Eq. (41), a system of the form studied in Section 4.2, with coefficients -1 and $-(a b)$. Hence, the analytical solution for this problem can be obtained from the analytical solution in Eqs. (34)-(35) in a straight forward manner. In this case, $u$ is discontinuous, whereas $p$ has a discontinuous derivative. The analytical solution on $[0, \pi)$ for both $u$ and $p$ with $\omega_{0}=16$ is shown in Figure 14 at time $t=0.16 \pi$.

Let us turn to the numerical results. The procedure is the same as in Section 4 . Again, due to the staggering, we use a grid refinement factor of 3 . We compute the error for each of $M=6$ shifts of $\bar{x}$ (Eq. (36)) in the maximum norm on $[0, \pi$ ), and then take the average. In the following we have used $N=480,1440$ and 4320 . Hence, $h=2 \pi / N$, and we set $k=0.8 h$.

First we keep $a$ and $b$ discontinuous. The error for $u$ and $p$ as a function of time, up to $t=0.48 \pi$ is shown in Figure 15. From the analysis, we expect a first order error due to the discontinuity. We can see this first order error best at early times. For the smallest $N$ at later times, the first order error term does not dominate the growing second order frequency dependent error, as was the case for the error for the original system, as shown in Figure 3. 

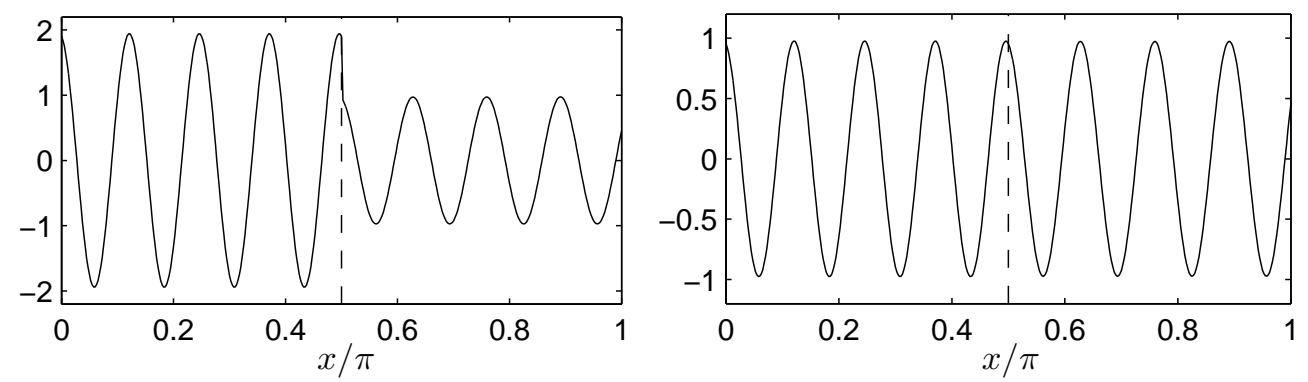

FIG. 14. The analytical solution of $u$ (to the left) and $p$ with $\omega_{0}=16$ is plotted on the interval $[0, \pi)$ at $t=0.16 \pi$.
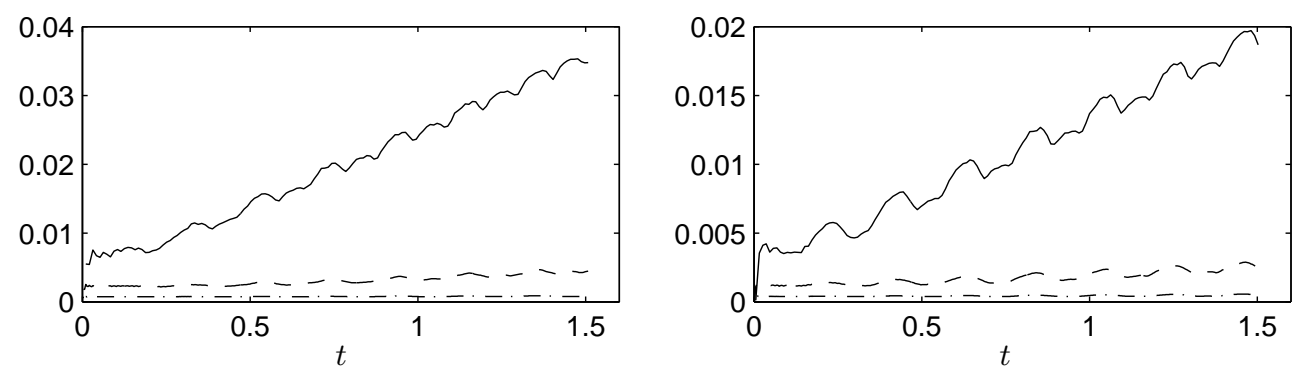

FIG. 15. Error in $u$ versus $t$ (to the left) and in $p$ versus $t$, for $N=480,1440$ and 4320, with $a$ and $b$ kept discontinuous.

We now repeat the same numerical experiment with $a$ and $b$ modified as defined in Eq. (47). The error in the numerical approximations of $u$ and $p$ in this case is plotted in Figure 16. Computing the convergence rates based on these results, we
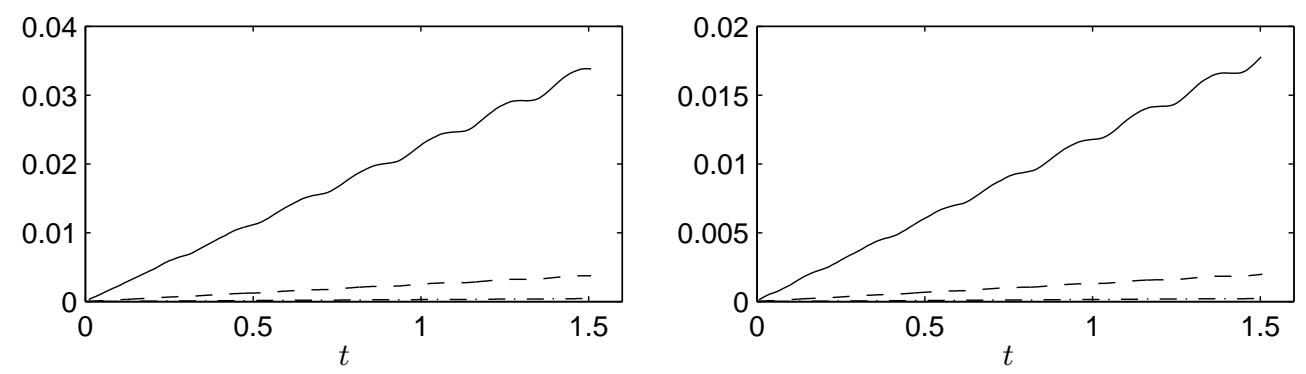

FIG. 16. Error in $u$ versus $t$ (to the left) and in $p$ versus $t$, for $N=480,1440$ and 4320 with a and $b$ regularized as given in Eq. (47).

find that they are very close to the predicted second order, as shown in Figure 17. That is, in this case both the error arising from the discretization at the discontinuity and the error that would arise for smooth coefficients also, are both second order.

Hence, by a simple modification of $b(x)$, we have obtained a second order accurate scheme for this problem which has a discontinuous solution $u$ and a continuous but non-smooth solution $p$.

7. Discontinuous material coefficients in 2D. Consider the system in Eq. (1). We assume that $a$ and $b$ are piecewise constant coefficients. We do not here consider the general case, but start by considering the special case where the 

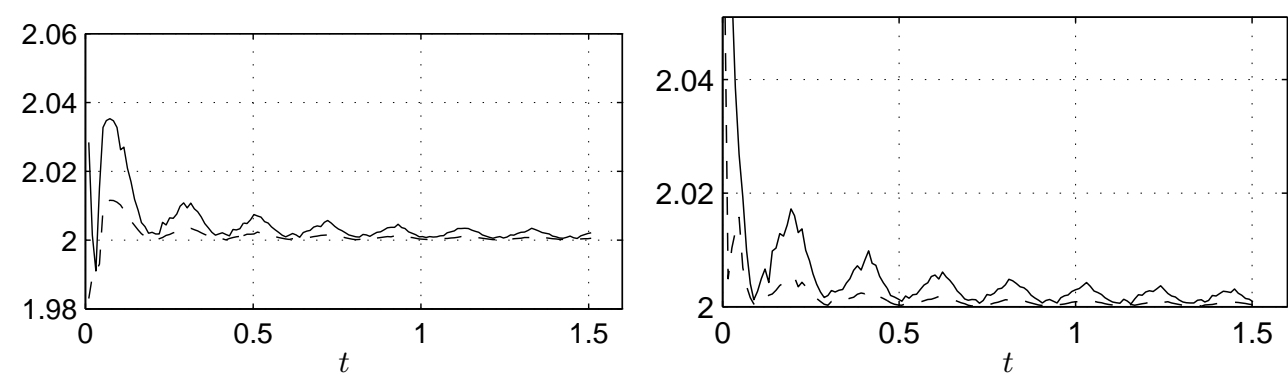

FIG. 17. The convergence rate for the numerical approximation of $u$ versus $t$ (to the left) and in $p$ versus $t$, as computed from the results displayed in Figure 5 (Based on the errors for $N=480$ and $N=1440$, solid line, and $N=1440$ and $N=4320$, dashed line).

discontinuities lie along the line $x=\bar{x}$. That is, we define $a$ and $b$ as

$$
a(x, y)=\left\{\begin{array}{ll}
a^{L} & \text { if } x \leq \bar{x}, \forall y \\
a^{R} & \text { if } x>\bar{x}, \forall y
\end{array} \quad b(x, y)= \begin{cases}b^{L} & \text { if } x \leq \bar{x}, \forall y \\
b^{R} & \text { if } x>\bar{x}, \forall y\end{cases}\right.
$$

The pressure $p$ is continuous across $\bar{x}$. For the velocities, the normal component is continuous, hence in this case, $u$ is continuous. The tangential component, $v$ in this case, is however discontinuous. We have the jump conditions

$$
[p]=p^{R}-p^{L}=0, \quad[u]=u^{R}-u^{L}=0, \quad\left[b^{-1} v\right]=v^{R} / b^{R}-v^{L} / b^{L}=0,
$$

which hold for all $y$ along $x=\bar{x}$. The third jump condition has been obtained from the first, using the equation $v_{t}=b p_{y}$ and the fact that we are considering a vertical boundary.

Moreover, if the velocity is irrotational initially $\left(u_{y}-v_{x}=0\right)$, then it will be so at all times. We will assume that this is the case. Using this, together with the system (1) and the jump conditions above, we find the following jump conditions for the derivatives,

$$
\begin{gathered}
{\left[a\left(u_{x}+v_{y}\right)\right]=0, \quad\left[b p_{x}\right]=0, \quad\left[p_{y}\right]=0} \\
{\left[u_{y}\right]=0, \quad\left[v_{x}\right]=0, \quad\left[b^{-1} v_{y}\right]=0}
\end{gathered}
$$

Combining the first and the last of these jump conditions, we get

$$
\begin{aligned}
& u_{x}^{R}=\frac{a^{L}}{a^{R}} u_{x}^{L}+\left(\frac{a^{L}}{a^{R}}-\frac{b^{R}}{b^{L}}\right) v_{y}^{L}, \\
& u_{x}^{L}=\frac{a^{R}}{a^{L}} u_{x}^{R}+\left(\frac{a^{R}}{a^{L}}-\frac{b^{L}}{b^{R}}\right) v_{y}^{R} .
\end{aligned}
$$

See also [16] for a discussion regarding these jump conditions.

7.1. Regularization for the Yee scheme. The unknowns are now the two velocity components $u$ and $v$, and the pressure $p$. We introduce a regular grid in two dimensions, with full and half coordinates in $x\left(x_{j}=j h, x_{j+1 / 2}=(j+1 / 2) h\right)$ and in $y\left(y_{j}=j h, y_{j+1 / 2}=(j+1 / 2) h\right)$. The grid points where $u$ and $v$ and $p$ are stored are all staggered in space, but the velocity components are stored at the same time level, as shown in Figure 18. We will use the extension of the definition of $D_{+}$and $D_{-}$ 


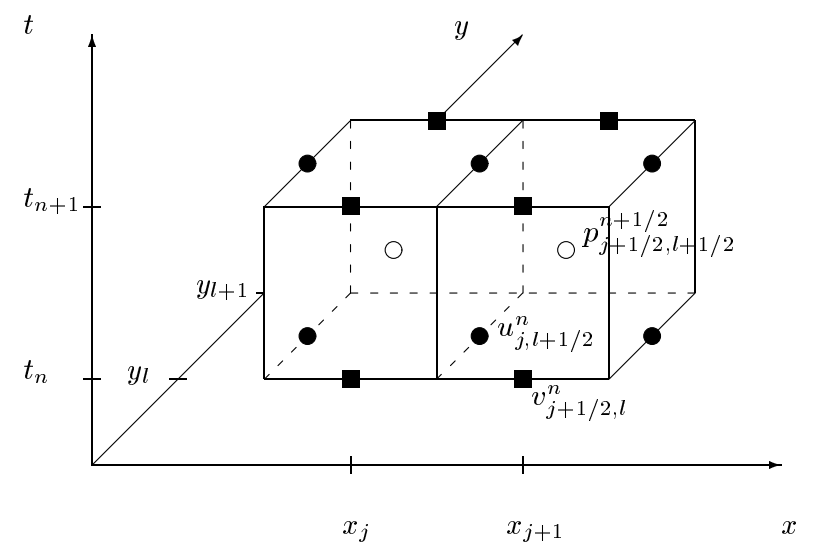

FIG. 18. The staggered grid.

as given in Eq. (8), to differences in the $x$ and $y$ direction, such that $D_{+x} u_{j, l+1 / 2}=$ $\left(u_{j+1, l+1 / 2}-u_{j, l+1 / 2}\right) / h$ and $D_{-y} p_{j+1 / 2, l+1 / 2}=\left(p_{j+1 / 2, l+1 / 2}-p_{j+1 / 2, l-1 / 2}\right) / h$, etc.

Consider the system in Eq. (1) as discretized by a generalized Yee scheme,

$$
\begin{aligned}
p_{j+1 / 2, l+1 / 2}^{n+1 / 2} & =p_{j+1 / 2, l+1 / 2}^{n-1 / 2}+k\left[a_{j+1 / 2, l+1 / 2}^{(1)} D_{+x} u_{j, l+1 / 2}^{n}+a_{j+1 / 2, l+1 / 2}^{(2)} D_{+y} v_{j+1 / 2, l}^{n}\right] \\
u_{j, l+1 / 2}^{n+1} & =u_{j, l+1 / 2}^{n}+k b_{j, l+1 / 2}^{(1)} D_{-x} p_{j+1 / 2, l+1 / 2}^{n+1 / 2}, \\
v_{j+1 / 2, l}^{n+1} & =v_{j+1 / 2, l}^{n}+k b_{j, l+1 / 2}^{(2)} D_{-y} p_{j+1 / 2, l+1 / 2}^{n+1 / 2} .
\end{aligned}
$$

We will need to apply different regularizations for $a^{(1)}, a^{(2)}, b^{(1)}$ and $b^{(2)}$. In the standard Yee scheme, $a^{(1)}=a^{(2)}=a$ and $b^{(1)}=b^{(2)}=b$.

To evaluate the pointwise accuracy of the spatial discretization, we can Taylor expand the right hand sides of the equations in the system in $x$ around $x=\bar{x}$, similarly to the one-dimensional case. Without regularization, we find that the discretizations in the first and second equation yields an $O(1)$ local truncation error in a band of points closest to the discontinuity. For the last equation, the stencil never crosses the discontinuity, and hence is not affected by it, and we get second order accuracy in all points.

To design a modification that allows only for first order local truncation errors closest to the discontinuity, with the goal of second order error in the numerical solutions for $u, v$ and $p$, we use the jump conditions in Eq. (51) in the Taylor expansions.

We suggest the following regularization: $a^{(1)}(x, y)=\tilde{a}(x)$, and $b^{(1)}(x, y)=\tilde{b}(x)$ with $\tilde{a}(x)$ and $\tilde{b}(x)$ as given in Eq.(18) and Eq. (22), respectively. Hence, this is the regularization we had also in the $1 \mathrm{D}$ case. However, we let $b^{(2)}(x, y)=b(x, y)$, with $b$ as defined in Eq. (49), i.e. we keep $b^{(2)}(x, y)$ discontinuous.

The coefficient $a^{(2)}(x, y)$ gets the most complicated definition. It is given as

$$
a^{(2)}(x, y)= \begin{cases}a^{L} & \text { if } x \leq \bar{x}-h / 2, \\ \Theta\left((x-\bar{x}) / h, a^{L}, a^{R}\right) \cdot \Phi^{L}\left((x-\bar{x}) / h, b^{L}, b^{R}\right) & \text { if } \bar{x}-h / 2<x \leq \bar{x}, \\ \Theta\left((x-\bar{x}) / h, a^{L}, a^{R}\right) \cdot \Phi^{R}\left((x-\bar{x}) / h, b^{L}, b^{R}\right) & \text { if } \bar{x}<x \leq \bar{x}+h / 2, \\ a^{R} & \text { if } x>\bar{x}+h / 2,\end{cases}
$$


where $\Theta\left(\eta, a^{L}, a^{R}\right)$ is defined in Eq. (19), and

$$
\begin{aligned}
\Phi^{L}\left(\eta, b^{L}, b^{R}\right) & =\frac{1}{2}\left(1+b^{R} / b^{L}\right)+\eta\left(b^{R} / b^{L}-1\right), \\
\Phi^{R}\left(\eta, b^{L}, b^{R}\right) & =\frac{1}{2}\left(1+b^{L} / b^{R}\right)+\eta\left(1-b^{L} / b^{R}\right) .
\end{aligned}
$$

The reason that the ratio $b^{L} / b^{R}$ enters this modification of $a$ is due to the mixed jump conditions in Eq. (52).

7.2. Numerical results. We solve the system (1) with $a(x)$ and $b(x)$ as given in (49) in the domain $[0,2 \pi] \times[0,2 \pi], 0 \leq t \leq T$. We consider a plane wave, impinging the discontinuity at an angle $\theta$. We set $a^{L}=b^{L}=-1$, and define

$$
\alpha^{L}=\cos (\theta), \quad \beta=\sin (\theta), \quad \alpha^{R}=\sqrt{1 /\left(a^{R} b^{R}\right)-\beta^{2}} .
$$

We then have the following solutions for $p, u$ and $v$ :

$$
\begin{aligned}
& p(x, y, t)= \begin{cases}\sin \left(\alpha^{L}(x-\bar{x})+\beta y+t\right)+\sigma \sin \left(-\alpha^{L}(x-\bar{x})+\beta y+t\right), & \text { if } x \leq \bar{x}, \forall y \\
\tau \sin \left(\alpha^{R}(x-\bar{x})+\beta y+t\right) & \text { if } x>\bar{x}, \forall y\end{cases} \\
& u(x, y, t)= \begin{cases}b^{L} \alpha^{L}\left(\sin \left(\alpha^{L}(x-\bar{x})+\beta y+t\right)-\sigma \sin \left(-\alpha^{L}(x-\bar{x})+\beta y+t\right)\right), \\
b^{R} \alpha^{R} \tau \sin \left(\alpha^{R}(x-\bar{x})+\beta y+t\right) & \text { if } x \leq \bar{x}, \forall y\end{cases} \\
& v(x, y, t)= \begin{cases}b^{R} \beta\left(\sin \left(\alpha^{L}(x-\bar{x})+\beta y+t\right)+\sigma \sin \left(-\alpha^{L}(x-\bar{x})+\beta y\right.\right. \\
\left.b^{R} \beta \tau \sin \left(\alpha^{R}(x-\bar{x})+\beta y+t\right)\right) & \text { if } x \leq \bar{x}, \forall y\end{cases} \\
&
\end{aligned}
$$

where the reflection and transmission coefficients are given by

$$
\sigma=\frac{b^{L} \alpha^{L}-b^{R} \alpha^{R}}{b^{L} \alpha^{L}+b^{R} \alpha^{R}}, \quad \tau=\frac{2 b^{L} \alpha^{L}}{b^{L} \alpha^{L}+b^{R} \alpha^{R}} .
$$

Below, we present some results for $a^{L}=b^{L}=-1.0, a^{R}=-0.2, b^{R}=-0.5$, where we let $\theta=\pi / 12$. In Figure 19, the analytical solutions for $u, v$ and $p$ at $t=0.3 \pi$ are shown.

We impose periodic boundary conditions on the outer boundary. These are not the correct boundary conditions, and disturbances will hence arise at the boundary. In the tests below, we run up to $T=0.3 \pi$, and we compute all errors in the maximum norm over the domain $\bar{\Omega}=[\pi / 2,3 \pi / 2] \times[\pi / 2,3 \pi / 2]$, as denoted by $\|\cdot\|_{\bar{\Omega}, \infty}$. We do so for each of $M=6$ shift of $\bar{x}$ around $\bar{x}=\pi$ (in analogy with Eq. (36)), and then compute the average. With $N$ grid points in both directions, we have $h=2 \pi / N$, and we set $k=0.6 h$.

We start with the case of no modification, i.e. we keep both $a$ and $b$ discontinuous, as defined in Eq. (49). In Figures 20-21, the errors in $u, v$ and $p$ are plotted versus $t$. The errors are absolute errors. The magnitude of the error in $v$ is smaller, but so is the norm of $v$. The numerical errors indicate a first order convergence (remember the refinement factor of 3 ).

We now modify $a$ and $b$, using the suggested modifications in and above Eq. (54) in the discretization (53). We consider the error in $u, v$ and $p$ as a function of time, for 


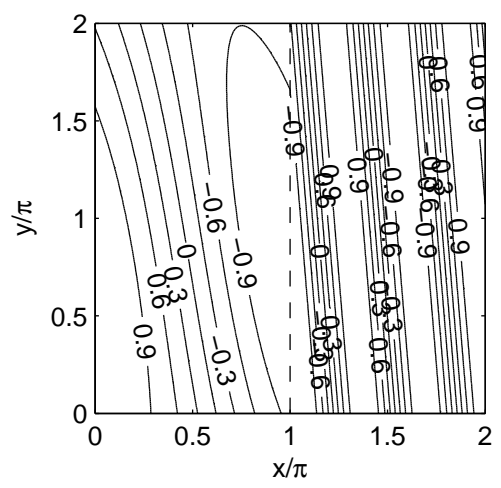

(a) $u,\|u\|_{\infty}=1.20$.

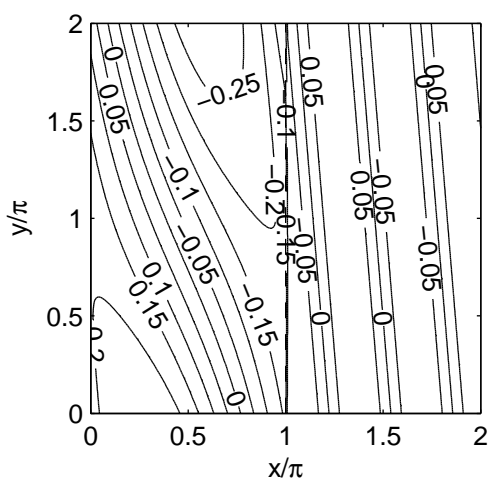

(b) $v,\|v\|_{\infty}=0.29$.

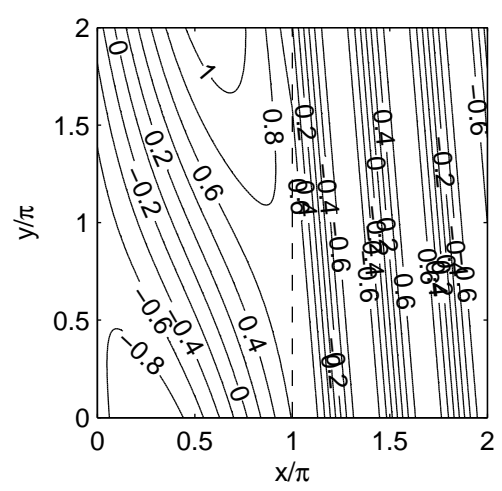

(c) $p,\|p\|_{\infty}=1.12$.

FIG. 19. The analytical solution for $u, v$ and $p$ at $t=0.3 \pi(\bar{x}=\pi)$. For $x<\bar{x}$, both the incoming and reflected wave contribute to the solution. For $x>\bar{x}$, there is only the transmitted wave.
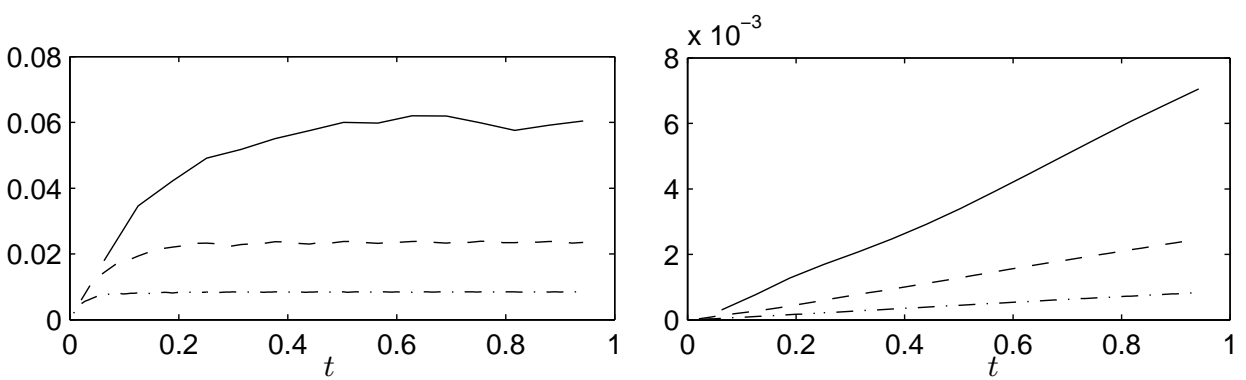

FIG. 20. Error in maximum norm for $u$ (to the left) and for $v$ plotted versus $t$ for $N=60,180$ and 540, with $a$ and $b$ kept discontinuous.

the three grid resolutions $N=60,180$ and 540. In Figure 22, the errors for $u$ and $v$ are plotted versus time $t$. The error for $p$ is plotted in Figure 23. These results are to be compared to the errors plotted in Figures 20-21, with $a$ and $b$ kept discontinuous. We can note that by introducing the modification of $a$ and $b$, the errors are significantly smaller than without the modification. The computed convergence rates are close to second order. 


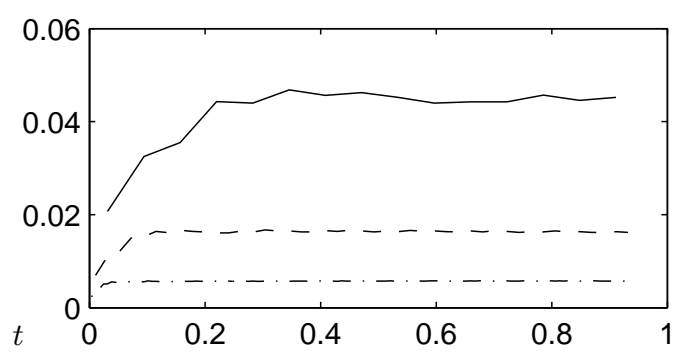

FIG. 21. Error in maximum norm for $p$ versus $t$ plotted up to $t=0.3 \pi$ (as in Figure 20 but for $p)$.
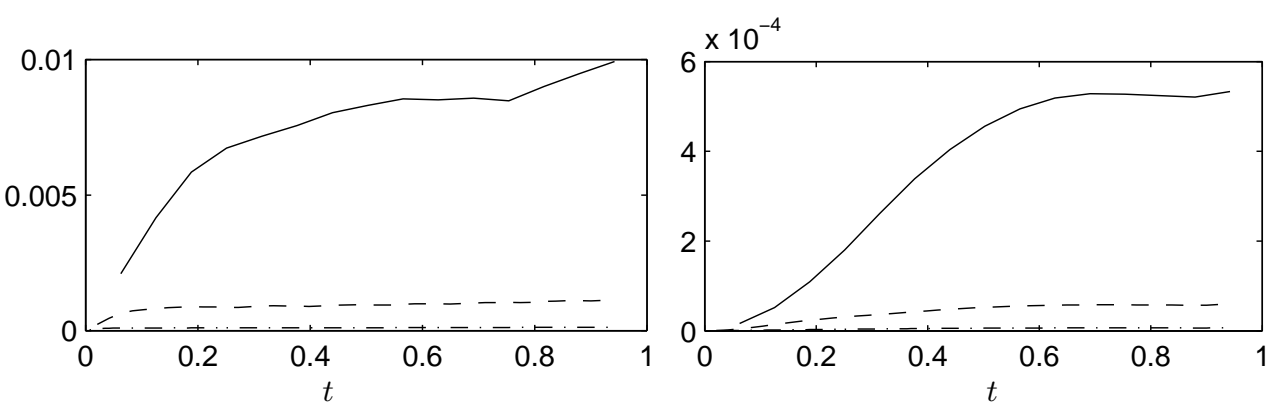

Fig. 22. Error in maximum norm for $u$ (to the left) and for $v$ versus $t$, for $N=60,180$ and 540. Same as in Figure 20 but with $a$ and $b$ modified as given in and above Eq. (54).

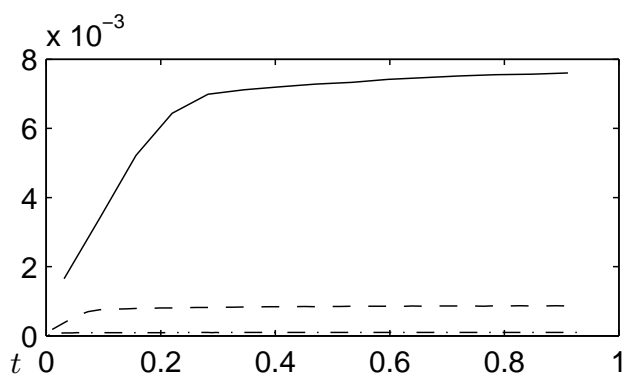

FIG. 23. Error in maximum norm for $p$ versus $t$ (As in Figure 22, but for $p$ ).

8. Conclusions. In this paper, the efficient Yee scheme for numerical approximation of wave propagation is generalized to handle discontinuous material coefficients. We improve the convergence properties by modifying these coefficients near the material interfaces.

In one space dimension we prove that the original Yee scheme gives a first order numerical approximation when the interface is located at an arbitrary point relative to the grid. The new modified scheme is proved to be of second order. This technique is also applied to a discretization with the Yee scheme structure but with a wider stencil in space, which gives fourth order accuracy in both space and time for smooth material coefficients. The higher order discretization can be written as the Yee scheme with added correction terms. By modifying the coefficients close to the interface we reach overall second order accuracy even for discontinuities. The phase error is still of fourth order, and the total error of the scheme has in numerical experiments been shown to be much lower than for the Yee scheme. 
A problem with discontinuous solution is also studied. Even in this case the coefficients in the Yee scheme can be modified near the interface to give second order accuracy. Discontinuous solutions are relevant for problems in higher dimensions. The main advantages of our type of higher order interface approximations, compared to many others, are that the resulting schemes can be proved to be stable and that they have the same uniform staggered structure for the full computational domain including areas with interfaces. This structure is the basis for highly efficient computer implementations.

In the last section, a simple example for extension to two space dimensions is included. The derivation and implementation of approximations for arbitrary interfaces in two dimensions will be presented in a forthcoming paper.

\section{REFERENCES}

[1] G. Cohen And P. Joly, Construction analysis of fourth-order difference schemes for the acoustic wave equation in nonhomogeneous media, SIAM J. Numer. Anal., 33 (1996), pp. 1266-1302.

[2] A. Ditkowski, K. Dridi, And J.S. Hesthaven, Convergent cartesian grid methods for Maxwell's equations in complex geometries, J. Comput. Phys., 170 (2001), pp. 39-80.

[3] B. Gustafsson, The convergence rate for difference approximations to mixed initial boundary value problems, Math. Comp., 29 (1975), pp. 396-406.

[4] B. Gustafsson And E. Mossberg, Time compact high order difference methods for wave propagation, SIAM J. Sci. Comput., 26 (2004), pp. 259-271.

[5] B. Gustafsson and P. Wahlund, Time compact difference methods for wave propagation in discontinuous media, SIAM J. Sci. Comput., 26 (2004), pp. 272-293.

[6] B. Gustafsson And P. Wahlund, Time compact high order difference methods for wave propagation, 2-D, J. of Sci. Comput., 25 (2005), pp. 195-211.

[7] J.S. HeSthaVEn, High-order accurate methods in time-domain computational electromagnetics: A review, Adv. Imag. Electron Phys., 127 (2003), pp. 59-123.

[8] B. Lombard and J. Piraux, Numerical treatment of two-dimensional interfaces for acoustic and elastic waves, J. Comput. Phys., 195 (2004), pp. 90-116.

[9] J. Piraux and B. Lombard, A new interface method for hyperbolic problems with discontinuous coefficients: one-dimensional acoustic example, J. Comput. Phys., 168 (2001), pp. 227-248.

[10] A. Taflove and S.C Hagness, Computational Electrodynamics: The Finite Difference Time Domain Method, Artech House, 3rd edition, 2005.

[11] A.-K. ToRnBerg, Regularization techniques for singular source terms in differential equations, in A. Laptev, editor, European Congress of Mathematics (ECM), Stockholm, Sweden, June 27-July 2, 2004. Zurich: European Mathematical Society, 2005.

[12] A.K. Tornberg And B. Engquist, Regularization techniques for numerical approximation of PDEs with singularities, J. of Sci. Comput., 19 (2003), pp. 527-552.

[13] A.K. Tornberg And B. Engquist, Numerical approximations of singular source terms in differential equations, J. Comput. Phys., 200 (2004), pp. 462-488.

[14] A.K. Tornberg, B. Engquist, B. Gustafsson, and P. Wahlund, A new type of boundary treatment for wave propagation, BIT, 46:s145-s170.

[15] K. S. YeE, Numerical solution of initial boundary value problems involving Maxwell's equations in isotropic media, IEEE Trans. Antennas Propag., 14 (1966), pp. 302-307.

[16] C. Zhang and R.J. LeVeque, The immersed interface method for acoustic wave equations with discontinuous coefficients, Wave Motion, 25 (1997), pp. 237-263.

[17] S. ZhaO AND G.W. WeI, High order FDTD methods via derivative matching for Maxwell's equations with material interfaces, J. Comput. Phys., 200 (2004), pp. 60-103. 
